\title{
Article \\ Cyber Forensic Review of Human Footprint and Gait-based System for Personal Identification in Crime Scene Investigation
}

\author{
Kapil Kumar Nagwanshi \\ Rungta College of Engineering and Technology Bhilai, India \\ * Correspondence: kapilkn@ieee.org; Tel.: +91-930-301-8770
}

\begin{abstract}
Human footprint is having a unique set of ridges unmatched by any other human being, and therefore it can be used in different identity documents for example birth certificate, Indian biometric identification system AADHAR card, driving license, PAN card, and passport. There are many instances of the crime scene where an accused must walk around and left the footwear impressions as well as barefoot prints and therefore it is very crucial to recovering the footprints to identify the criminals. Footprint-based biometric is a considerably newer technique for personal identification. Fingerprints, retina, iris and face recognition are the methods most useful for attendance record of the person. This time world is facing the problem of global terrorism. It is challenging to identify the terrorist because they are living as regular as the citizens do. Their soft target includes the industries of special interests such as defense, silicon and nanotechnology chip manufacturing units, pharmacy sectors. They pretend themselves as religious persons, so temples and other holy places, even in markets is in their targets. These are the places where one can obtain their footprints easily. The gait itself is sufficient to predict the behaviour of the suspects. The present research is driven to identify the usefulness of footprint and gait as an alternative to personal identification.
\end{abstract}

Keywords: ANN; biometric; crime-scene; fuzzy logic; gait; human footprint; Hidden Markov Model; PCA; Recognition.

\section{Introduction}

The human brain recognize and classify objects, person, or places in an apparently efficient, quick and smooth manner. For example, a person can identify his childhood friend comfortably in an old group photograph without encountering any difficulty. Since the recognition process happens so automatic and fast, therefore, it is difficult to translate this behavior into computer algorithm as perfect as the human being. In other words, it is neither permissible to define nor to observe meticulously whence the recognition process acts. Everything in this world, from the universe to an atom like oxygen is a pattern. Water is a pattern of an assembly of hydrogen and oxygen; a human face is a pattern based on the contour of the eye, ear, hair, lips, cheek, etc. Likewise, the biometric oriented system is also a pattern based on biological matrices. The human footprint has an enormous legal capacity in Austria and some other countries such that one cannot ignore the role of biometric in society. Biometric passports are nowadays standard in India for travel across the world.Traveling by air is also very common; therefore, terrorists can take benefit of the vulnerable identification system available in railways, bus terminals or any such crowded spots to harm the assets of the nation. One must have a full-proof system which is capable of distinguishing people quickly to ensure the security of such stated places. Instead of the accomplishment of monetary transactions, the overpass of international borders and many more urgent solicitations, they are accountable for personal identification, which might be unavoidable.A traditional token-based method relies on the passport, credit or debit cards, or any such legal identity cards while the knowledge-based identification uses PINs, user's confidential personal information such as mother's maiden name, the city he born etc. gives a clue to find passwords, do not inherently imply the right capabilities. One may not be competent to at a guess that the person 
38 problem of information and personal security.

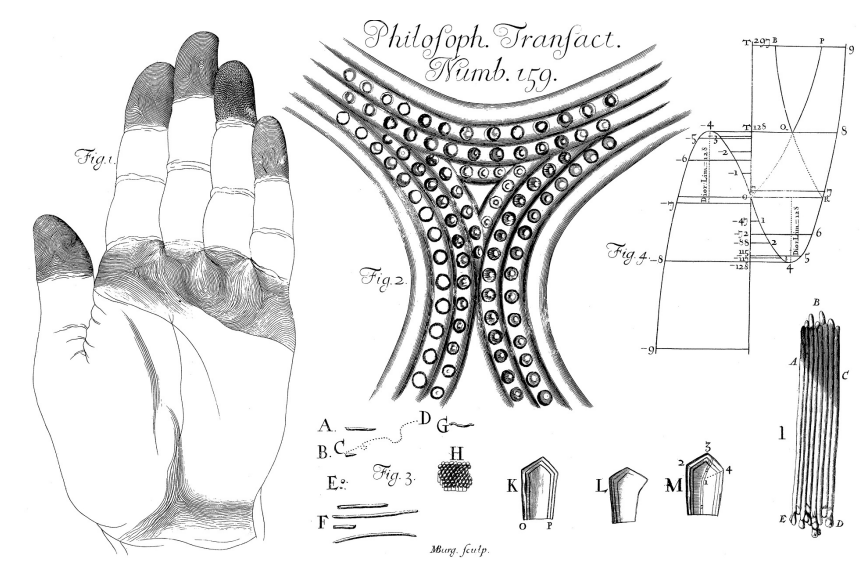

Figure 1. Friction ridge skin observations of human fingerprints.(Courtesy:[1])

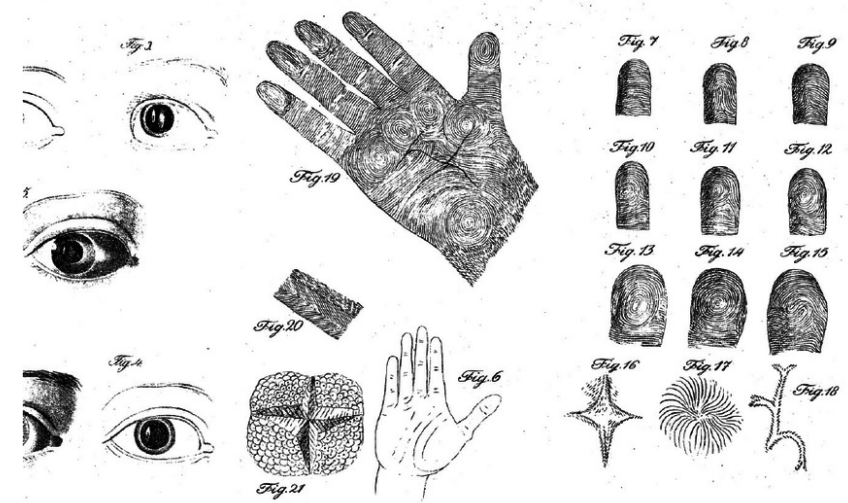

Figure 2. Engravings of fingerprints from Dissertation on "Physical Examination of the Organ of Sight". (Courtesy :[2])

Biometric based personal identification system is the traditional and most appropriate method for authentication, if we compare it to smart card based or password-based methods[3-7]. Fingerprint as a personal biometric proves it's capability as the signature from ancient time (around $1000 \mathrm{BC}$ ) used in different geographical locations Babylon pressed the tips of their finger into clay to record business transactions, China used ink-on-paper foot and finger impressions for business and to help identify their children, Nova Scotia, and Persia. On the epidermis portion of the body for example finger, palm, the sole, and toe determine by different friction ridge lines present on it. These friction ridges make an impression on surfaces such as glassware, plastic articles, paper sheet, and wooden items. Ref. [1] give a profound observation on friction ridge skin of the finger, palm, and foot (see Figure 2 ). In the year 1686, Macello Malpighi observed loops, whorls, arches, and ridges in fingerprints as the unique pattern for identification. In Figure 2, Ref. [2] documented nine distinct patterns to help identify types of prints. Regardless of this invention, the use of fingerprints did not catch on then.

In the year 1858 Figure 3 shows a first legal contract between Herschel, a British Administrator of West Bengal, and Rajyadhar Konai, a local businessman, first used fingerprints on domestic procurement. In the year 1901 Henry, an Inspector General of Police in Bengal, India, had developed the first system of classifying fingerprints. This system had later adopted as the official system by many countries[8]. 


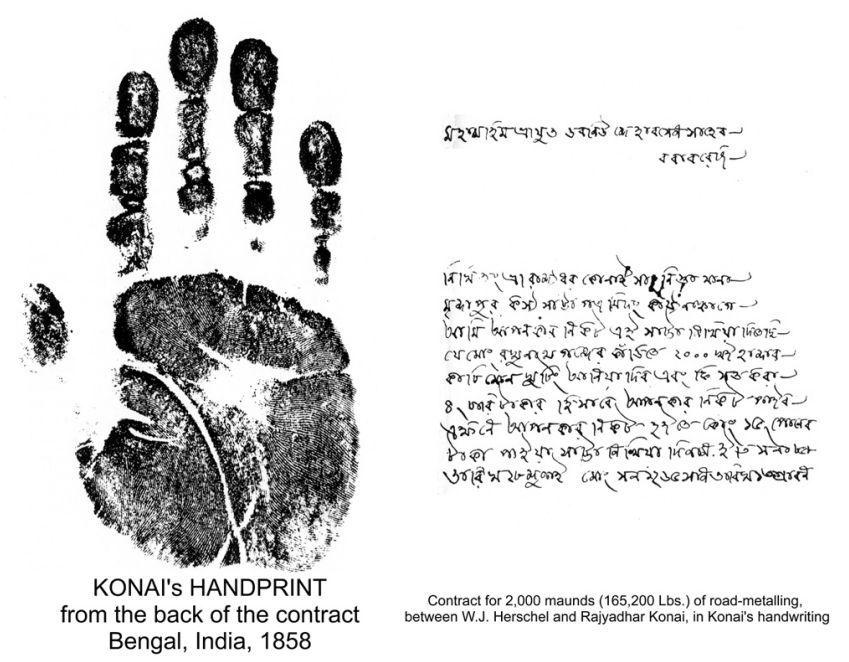

Figure 3. The India was the first country who adopt fingerprint in a legal capacity in the year 1858 . This imprint of the document shows first legal civil contract use of palm-print as a signature.

The major function of biometric to verify and identify a genuine person or importer as tools of surveillance that scan for terrorist or unlawful activity for gaining access to a secret system. The automated system is necessary and conforms enhanced security standards despite technical limitations, but misuse of identity theft by criminals leads to hazards in the society. Fingerprints, face, and eye are the common biometric traits used commonly for attendance, in ATMs to withdraw money, to purchase new mobile connection or in driving license [3,9]. Interpol presentation authored by [10], presented historical background of biometric traits and its utility in crime scene investigation from the age of non-electricity time. In addition, with fingerprints, face and retinal images, palm prints has also been used in various security applications such as social security cards and passports [11-15]. Hand shape and texture can also be helpful in personal recognition but hand shape is not sufficient, hence another modality should be added to obtained the recognition pattern [16,17].

Ref. [20] had acutely examined for the individuality of human footprints, entire report on several physical characteristics of the individuals who made them was retrieved. The shape, or form, of an individual's foot, is unique in characteristics so no surprise to physical anthropologists. The combined effects of heredity and life experiences are operative in determining the size and shape of our feet, and for each of us, the appearance of those consequences is uniquely our own.

According to Ref. [21] forensic podiatry is defined as "the employment of in-depth and researched podiatry knowledge and expertise in forensic investigations, to confirm the relation of an individual with a crime scene, or to solve any other legal interrogation concerned with the foot or footwear that requires knowledge of the functioning foot". Forensic podiatrists support in the identification of perpetrators of crime where barefoot prints, footwear, and the CCTV gait proof are incorporated. The crime scene identification requires their expertise in the evaluation of the impressions of foot and lower limb use, the estimation, and matching of wear associated with the foot/shoe interface and in comparisons compelling evidence of shoe size. In their CCTV movie, forensic podiatrists examine the gait patterns of a person obtained on CCTV with suspected offenders. The identification of human remains from the comparison of the feet of the deceased with detail recorded in the podiatry reports of missing individuals requires forensic podiatrists. Figure 4 a shows footprint for determination of sex on the basis of BBAL-breadth of footprint at ball and BHEL-breadth of footprint at heel measurement. The HB Index has calculated as $(B H E L \div B B A L) \times 100$, for sex determination[18,22]. Figure $4 \mathrm{~b}$ shows different points for stature estimation from the anthropomorphic analysis of footprints[19]. The term "recognition in proposed work refers to both verification (1:1 comparison with a claimed identity 


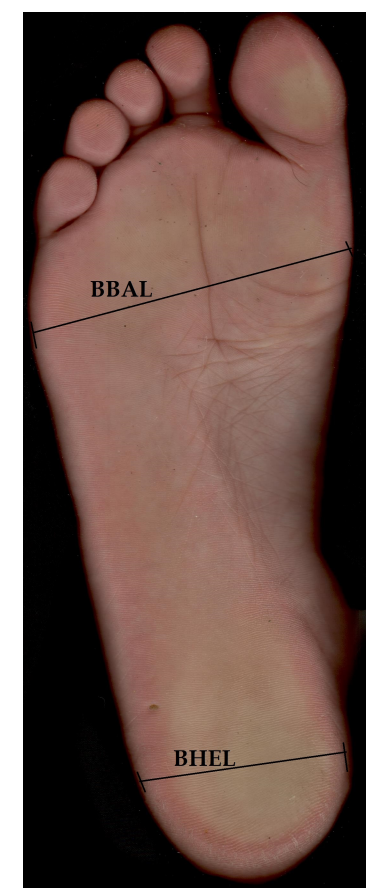

(a)

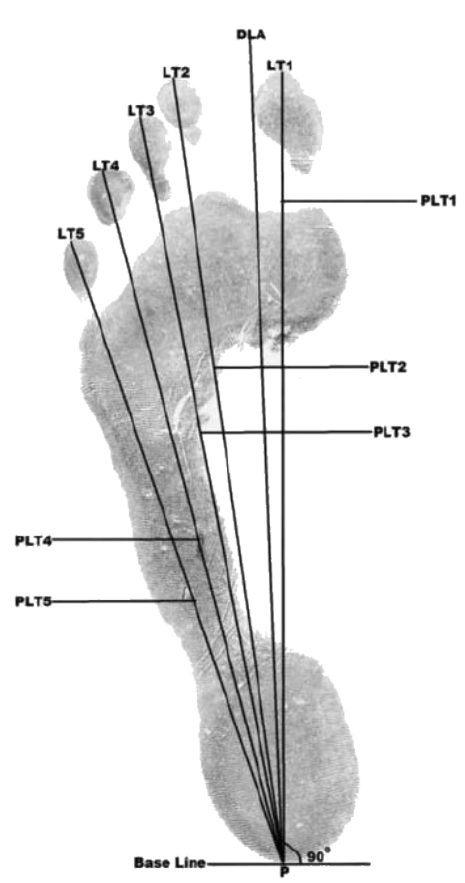

(b)

Figure 4. (a).Footprint showing the landmarks and measurements for BBAL-breadth of footprint at ball and BHEL-breadth of footprint at heel. The HB(Heel-Ball)Index of a footprint was derived as $(B H E L \div B B A L) \times 100$, for sex determination using footprint [18];(b). Landmarks and diagonal length measurements on left footprints, PLT1-PLT5(distance from P to the big toe-PLT1, index toe-PLT2, middle toe-PLT3, fourth toe-PLT4, little toe-PLT5 respectively), measurements taken from the mid-rear heel point $\mathrm{P}$ to the most anterior point of left toes LT1 to LT5 on footprints. DLA is the designated longitudinal axis[19].

88

template) and identification (1: n comparison to find a matching template, if existing), as is used in several literature"[7,9,23,24].

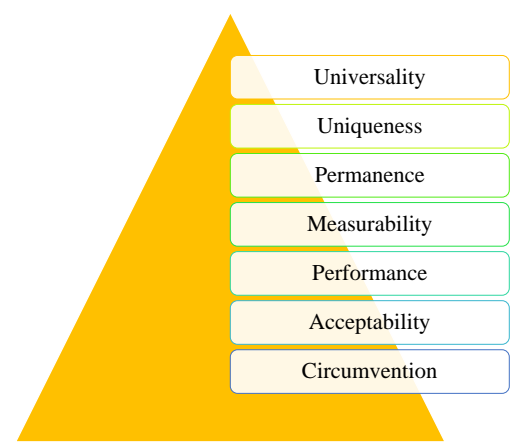

Figure 5. Trade-offs between biometric systems[25].

Figure 5 shows seven biometrics measures which have been discussed by Ref. [3,6,25] includes, " (i) The universality, is every subject in this system should hold the feature; (ii) these attained features must be effectively diverse for subjects in the applicable domain such that one can be distinguishable from another is uniqueness; (iii) the way in which a feature illustrates the discrepancy temporally is dealt with the permanence problem, and it is expected that the permanence will be reasonably invariant over 
time for the specific biometric matching algorithm; (iv) measurability or collectability is the simplicity of attainment or capacity of the feature that permits further processing and extraction of the applicable feature sets; (v) performance is the measurement of accuracy, speed, and robustness of algorithm used; (vi) acceptability is the measurement of acceptance of the technology with respect to the biometric feature captured and arbitrated, and, (vii) circumvention denotes to the ease with which a trait might be counterfeit. No single biometric system from physiological to behavioural meet all the requirements of each promising application. "

\section{Motivation}

Ref. [26] presented an analysis of foot and footwear characteristics, impressions, and track ways that lead to significant evidence in a crime scene investigation. Ref. [27-29] have implemented a system based on eigenfeet, ballprint and foot geometry for the small dataset of 16 entries. Ref. [26], have published a criminological and analytical study on footprint and footwear. Ref. [30], had applied footprint and foot outline dimensions for a particular tribe of criminal background which helps in an estimation of the build of a criminal. His study also signifies the utmost importance of footprint into understanding the crime scene investigations. Ref. [31] give a model based on neural networks and fuzzy logic with a claimed recognition rate of $92.8 \%$. Ref. [32-36] introduce modified sequential haar energy transform technique, and PCA for recognition. Ref. [37] has extracted fingerprint minutiae feature and PCA footprint features of newborn on Raspberry Pi system. Ref. [38] exhibit the use of PCA and Independent Component Analysis (ICA) for footprint recognition. Ref. [39] has used ANN for the the feature extraction and recognition with a recognition rate of $92 \%$. Primarily based on gap evaluation following are the motivations to propose a new approach to locating the uniqueness of human footprint. (a) Personal identification using gait is very difficult as it is based on behavioral patterns. It could be used for behavioral analysis of a person, but not for personal recognition. In Ref. [40] used highly correlated data (as all the images were collected in a day) that gives $100 \%$ recognition rate with only 5 subjects by using shoe-type sensor could be a significant constraint in the view of users. Ref. [41] and Ref. [42] has also done experimentation for gait based recognition, but addition of more subject lead to degradation in performance. (b) In Ref. [43], used Euclidean distance based personal identification among 10 persons with a recognition rate of $85 \%$ using normalized static footprint where user ought to make stand up stance each time. This was very basic method give direction to implement more realistic approach for footprint based personal identification. (c) Personal identification based on footprint cannot be use as a method for attendance monitoring, or biometric based transactions. On the other hand security of public places for example hospital, airport, religion places, nanotechnology based hi-tech industries etc. is a challenging task. To overcome from this problem footprints are very important to use in legal capacity to capture as other biometric modalities such as fingerprints, palm-prints, and retina images. (d) Number of subjects taken by other footprint based methods are limited to very few subjects. This motivates to add more number of subjects. Very limited number of subjects say ten exposed in most of the methods for footprint identification. Proposed method will utilize the fuzzy logic based method for personal identification. Considerably more than 300 subjects with the temporal aspect will be taken into account to fill the gap identified in existing methods.

\section{Review of Systems}

Despite installed CCTV it is very hard to identify a crime suspect who wears masks in face and gloves on hand, but he was not able to hide his footprint at crime scene walking pattern or gait behavior[44]. Analysis of recorded gait pattern compared with the gait pattern of a suspect and was convicted and sentenced to prison. Forensic podiatrists solved a lot number of cases based on video footage of criminals and the available footprints from the spot. In the year 1862 barefoot print of Jessie McLachlan convicts her in the crime scene of a woman's murder [45].In a murder case at Waushara County, Wisconsin, sock-clad footprints appeared on the motel room floor encompassing the slain 
body of Robert Kasun. After the detailed analysis of the footprint and its comparison with prime suspect Petersen convicted a life sentence without parole[46]. Now it is very standard practice to check for foot related pieces of evidence, and it proposed the necessity of legal podiatry organization. Robert Kennedy of the Royal Canadian Mounted Police (RCMP) researched on the uniqueness of footprints. Started in the year 1990s with a collection of 24,000 footprints. The collection proves the hypothesis of the unique character of footprints. From a rigorous statistical analysis of the footprint data, Kennedy has concluded the chances of matching of the footprint is one in 1.27 billion[46-48]. The American Society of Forensic Podiatry formed in the year 2003 by founder John DiMaggio with fifty-one members from different universities and research organizations. Most of the available literature focused on crime scene investigation[49]. The forensic podiatry discipline focused on three main application areas: (a) Footprints (This is principle area of discussion in present work), (b) Gait Analysis based on CCTV footage, and (c) Footwear. Previous section discuss the background and historical development of biometric system such as fingerprints, palmprints, and finally footprints. Present section gives comprehensive literature survey on gait based recognition system, footprint based recognition system, fuzzy logic, and analytical tools BigML ${ }^{\circledR}$ and IBM $^{\circledR}$ Watson Analytics.

\section{Gait-based system}

At the time of Aristotle ( $382-322$ BCE ), some evidence proves the applicability of gait examination. One interesting thing about India is the gait analysis of any person to see his/her characteristics for suitability of marriages. Giovanni Borelli (1608 - 1679) uses first scientific gait analysis research [50].

\subsection{Body Shape and Gait}

According to Ref. [51], a gait is determined using person's four main walking stances. They used viewpoint based template matching of body silhouettes captures height, width, and part proportions of the human body(silhouette extraction), on the other side cyclic gait capture stride length and the amount of arm swing analysis to extract keyframes (identify key frames) to compare with the training frames (template matching). The frame matching gives statistical parameters and matching score of two templates $t_{1}$ and $t_{2}$ as eq. 1

$$
C\left(t_{1}, t_{2}\right)=\frac{\max \left(\hat{t_{1}} \times \hat{t_{2}}\right)}{\max \left(\hat{t_{1}} \times \hat{t_{1}}\right) \times \max \left(\hat{t_{2}} \times \hat{t_{2}}\right)}
$$

where $\hat{t}$ is given by eq. 2

$$
\hat{t}=\frac{(t-\operatorname{mean}(t))}{\sigma(t)}
$$

and $\times$ is cross correlation operation. The maximum value of $C\left(t_{1}, t_{2}\right)$ is chosen. They used datasets from the MIT database shows 25 subjects (14 male, 11 female), the CMU MoBo database motion sequences of 25 subjects ( 23 male, two female) exercising on a treadmill, the U.Maryland database with 55 individuals (46 male, nine female), and the University of Southampton database contains 28 subjects walking indoors on track. Match scores for the across-gait condition vary from $76 \%$ to $100 \%$ on different profile test conditions.

\subsection{Invariant Gait Recognition}

Ref. [52] presents an invariant gait recognition approach. Human gait is spatiotemporal in nature. This algorithm gives a comprehensive solution to synthesizing arbitrary views of gait and applying incorporated views for gait recognition at any arbitrary angle to the camera on a perspective plane. Their dataset consists of 12 subjects, walking onward straight lines at varying values of azimuth angle $\theta$ from $0^{\circ}, 15^{\circ}, 30^{\circ}, 45^{\circ}$ and to $60^{\circ}\left(G_{0}, G_{15}, G_{30}, G_{45}\right.$, and $\left.G_{60}\right)$. Initially, it starts with capturing original video for estimating the azimuth angle; subsequently, the video sequence synthesized in a new view. 
A simple, and accurate, camera calibration procedure was also given. This algorithm uses very less number of subjects which is not sufficient to use in real life for person recognition. The recognition rate, FMR, FNMR, FRR, and so forth has not quoted in the literature.

\subsection{Hidden Markove Model in gait}

Ref. [53],shows the advanced research on identification by observation of gait and its to prove that his system is realistic which may likely to be developed and used in the years to come. Unobtrusiveness is the feature in which the prior consent is not required to capture the gait. The clothes, walking surface, walking speed, and emotions affects the gait pattern. Pattern ambiguity is a possibility in several cases, for example, the same kind of person (considering emotions) may have the same gait pattern. Adding another modality like the foot, retina, or palmprint (obtrusive) in conjunction with gait can make this identifiable. But if we add pattern it will need personal consent while foot pressure (requires special instruments to capture) or the face can help significantly. The calculation of two frequency templates (Fourier examination determines harmonic component) is straightforward. On the other hand in spatial template matching the sequence of gait features must be compared with another sequence. If $T 1$ and $T 2$ are the fundamental walking periods then the cumulative distance is calculated by eq. 3 :

$$
D_{12}=\frac{1}{U} \sum_{t=1}^{T} u(t) D\left(f_{1}\left(w_{1}(t)\right), f_{2}\left(w_{2}(t)\right)\right)
$$

where $w_{1}(t)$ and $w_{2}(t)$ are the wrapping functions, and $U$ is given by eq. 4

$$
U=\sum_{t=1}^{T} u(t)
$$

and $D(\cdot)$ gives the distance between feature vectors at time $t$. HMM is applied in gait stances. For a model $\lambda$, and test feature vectors $\tilde{f}_{i}$, the higher probability is acknowledged to be identical to the test subject of one of the models associated with the database with $N$ number of subjects sequences is given by eq. 5

$$
i \operatorname{dentity}(i)=\underset{j}{\operatorname{argmax}} P\left(\frac{\tilde{f}_{i}}{\lambda_{j}}\right), \forall j=1,2, . ., N
$$

They manage 71 subjects in their database. The right subject gives high confidence in top 10 matches. Gait alone is not sufficient to provide reliable and accurate recognition; this gives clear implication to use gait in conjunction with other biometrics traits for best performance in personal identification. Gait-based method has been adopted by many researchers. But gait based technique is not full proof hence it is needed to add some other biometric trait such as the face. In Japan and France health-care personals are using gait to diagnose the problems that can be modeled with walking or fall pattern of a person Ref. [54,55]. Walking pattern and stepping pattern also known as dynamic footprint has been used to characterized biometric user identification. Principal component analysis is applied to identify the user with extracted features [42]. Ref. [56,57], has applied load distribution sensor based on fuzzy distribution to capture foot pressure from 30 volunteers with an estimated recognition rate of $86 \%$. Pressure sensor based system using neural network has observed $28.6 \%$ false acceptance rate [58]. Tivive et al.[59] presented a technique based on radar Doppler spectrograms for gait classification denoted in the time-frequency domain. Sensor quality is having a high impact on biometric based system for recognition as it should be measurable, distinctive, and permanent over time [60]. Ref. [61],developed a gait based recognition system and obtained unique dynamic plantar pressure patterns among 104 individuals. 


\subsection{View Transformation Model in the Frequency Domain}

Ref. [62] uses a total of 719 gait sequences from 20 subjects for gait recognition from 24 perspective directions at every 15 degrees using a view transformation model (VTM) in the frequency domain features to generate the training set of multiple subjects from features of one or a few of the view directions. Initially, the gait of a person is extracted from IR camera using temperature based background removal to construct an amplitude spectra for spatiotemporal gait silhouette volume(GSV) of a person scaling and registering the silhouette images. Gait periodicity extracts the frequency-domain features of the volume by Fourier analysis to generate training and test set for recognition. The verification rate at a false positive rate is $10 \%$ in the ROC (Receiver Operating Characteristics) curves and the averaged verification rate. These systems achieve higher performance than the earlier perspective projection (PP) system proposed by Ref. [52], except for the frontal view $G_{0}$.

\subsection{Gait for Fall Prevention}

Ref. [55] develops a technique of gait detection and a balanced signature extracted from a force plate to fall prevention in old aged person in the PARAchute project. Every year in France more than 9000 death tolls due to the falling of the senior citizens, which is the biggest medical challenge. Their system is quite simple and does not require the third person to monitor the elderly without intervening the daily life hence privacy is preserved. It can be function independently as a part of the home security network. The gait is analyzed based on video camera sequencing, while the balance is assessed using a small force plate. The force plate measures center of pressure (COP) displacement in anteroposterior $(\mathrm{AP})$, mediolateral (ML), and resultant (RD) directions to describing the static and dynamic stability with no limitation on the posture of the person. The obtained gait sequence enables the calculation of kinematic and spatiotemporal variables already identified as appropriate in an examination of the risk of falling.For better results, this system requires large dataset as well as data fusion of home based system and the clinical data.

\subsection{Floor Pressure Sensing and Analysis}

Ref. $[63,64]$ employs 3D trajectories of the center of foot pressures atop a footstep include both the single dimensional pressure silhouette and 2D position trajectories of the COP taken by a large area high-resolution pressure sensing floor. A set of features has extracted based on the 3D COP trajectories can be further used for personal identification together with other features such as stride length and cadence. The Fisher linear discriminant classifies the foot pressure. This approach has tested for a floor pressure dataset collected from 11 subjects in different walking styles, including varying speed walking and free style walking yields identification results with an average recognition rate of $92.3 \%$ and FAR of $6.79 \%$.

\subsection{Radar Doppler Spectrograms Gait Classification}

Ref. [59]introduces a radar Doppler image classification approach, for human gait based following the time-frequency domain from five subjects moving towards and away from the radar by $0^{\circ}$ and $30^{\circ}$ aspect angle including the nonobstructed line of sight. The initial two stages of this method are intended to extract Doppler features that can adequately describe human motion based on the nature of arm swings as free-arm swings, one-arm confined swings, and no-arm swings, and the third stage performs multi-layer perceptron (MLP) classification. The one-arm confined swings and no-arm swings can depict that individual carrying objects or a he is in stressed conditions. Maximum classification rate obtained was $91.2 \%$ in two arm motion, but the number of subjects taken was very less for a practical purpose. 


\subsection{Clothing-invariant Gait Identification}

Ref. [65] uses part-based clothing categorization and adaptive weight control for the identification of gait. It is tough to identify a gait while variations in clothing alter an individual's appearance due to change of the pattern of silhouettes of certain parts hence a part-based procedure has the implied on selecting the relevant parts of a gait. The clothing used in the data set of 48 subjects both male and female are Regular pants, Half shirt, Casual wear, Baggy Pants Full shirt, Raincoat, Short Pants Long Coat, Hat, Skirt, Parker, Casquette Cap, Casual Pants, Down Jacket and Muffler. The human body has divided into eight sections viz neck, waist, knee, pelvis, waist-knee, neck-knee, neck-pelvis, and pelvis-knee with four overlapping and the larger parts have a higher discrimination capability, whereas the smaller parts are also seeming to be unaffected by clothing alterations. Some clothes are common to different parts can be classified in a collective group. This technique exploits the discrimination capability using an equal weight for each part and controls the weights adaptively meant on the distribution of distances between the test set with the data set. The EER varies from 0 to $0.5 \%$ depending on clothing combination.

\subsection{Geometric Features and Soft Biometrics based Gait Recognition}

Ref. [66]introduced a new framework for gait recognition expanded with soft biometric information acquired as height and stride length information has employed in a stochastic framework for the detection of soft biometric features of high discrimination ability. The captured gait sequences are processed to extract the geometric gait features based on Radon transforms and on gait energy images and the soft biometric features. Ref. [67] utilized the calibrated stereoscopic sequences based on real-world coordinates and absolute distances captured in HUMABIO and ACTIBIO databases for the estimation of height and the stride length soft biometric features. These sequences trivially overcome in the determination of the features that resemble the highest-lowest part of the subject, concerning height, and to the largest distance between the legs in a gait cycle. Different characteristic plots in the paper demonstrate the efficiency of their approach applying various algorithms for gait feature extraction and different databases.Four curves have shown on each graph corresponding to the cumulative matching scores (CMS) using the gait feature (gait) only or coupled with height (gait+height), or stride length (gait+stride), or both soft biometrics (gait+height+stride). On extending the gait feature with added soft biometric information significantly enhances the gait recognition efficiency. This method reduces the FAR and FRR in the EER point from $4 \%$ to $15 \%$ decrement in the ACTIBIO database depending on the soft biometrics applied.

\section{Footprint-biometric recognition}

This section covers detailed literature survey of a footprint based biometric technique. Some of the literatures are intended to use the pressure sensing technique while others are based on optical sensing and imaging technique.

\subsection{The BIG-MAT system}

Ref. [43] used the BIG-MAT on the hard floor of size $440 \times 480 \mathrm{~mm}^{2}$ with the load cells forming the matrix of $10 \times 10 \mathrm{~mm}^{2}(44 \times 48$ cells $)$ to acquired total 110 samples from ten volunteers of the pressure distribution of footprints. An image of 256 gray levels at a sampling speed of $30 \mathrm{fps}$ for $5 \mathrm{sec}$ acquire the barefoot prints. The monochrome image is given by eq. 6 :

$$
f(x, y):-X / 2<x \leq X / 2,-Y / 2<y \leq Y / 2
$$

represents the pressure distribution of a footprint. (where $X$ and $Y$ are widths of the mat). Initially, in pre-processing phase, they use raw footprint to translation motion of the whole image to the center of mass then go for segmentation of in the form of separation of both feet. Followed by calculation of the center of the foot for both the feet. Then normalize the image on the center of mass. Finally, 
reconstructed whole footprint image. In recognition phase, they use the distance $\tau$ between the center of mass of both footprints and the angle $\theta$ between left and right footprints before normalization for personal identification. Product of the first eigenvector of the left $e_{L}$ and right $e_{R}$ footprints calculates the angle using eq. 7.

$$
\theta=\cos ^{-1}\left(\left(e_{L} \cdot e_{R}\right) /\left(\left\|e_{L}\right\|\left\|e_{R}\right\|\right)\right)
$$

Employment of geometric elements $\theta$ and $\tau$ for recognition gives evaluation function as shown in eq. 8 .

$$
\begin{array}{r}
d=\sqrt{\sum_{(x, y)}\left\{I_{p}(x, y)-I_{\tau_{n}}(x, y)\right\}^{2}} \\
\quad+\alpha\left|\tau_{p}-\tau_{r_{n}}\right|+\beta\left|\theta_{p}-\theta_{r_{n}}\right|
\end{array}
$$

where $p$ is input footprint and $r_{n}$ is the $n^{\text {th }}$ registered footprint $(\forall n \mid n=1,2, . ., N)$, and $N$ be the total number of subjects. The first term is the Euclidean distance between $I_{p}(x, y)$ and $I_{\tau_{n}}(x, y)$. The second and third terms are the absolute difference of the distance $\tau$ and the angle $\theta$ between the registered and input footprint before normalization. $\alpha$ and $\beta$ are weighting parameters. After comparison of the input to all registered footprint, the minimum $d$ recognize entered footprint as registered footprint. $85.00 \%$ is the highest recognition rate achieved, which appears to be inadequate for practical uses. The author intends to apply personal identification for automatic data acquisition for people when many family members live together.

\subsection{The Pressure Mat using HMM}

Ref. [40,68,69], proposed an unconstrained person identification technique using dynamic footprint. They use one-step walking data through a mat-type pressure sensor to acquire quantized COP (Center of Pressure) trajectory from eight subjects. Like [43], they calculate the width $w_{x}$ and $w_{y}$ of the sensor, in the same way by using eq. 9 (used $w_{x}$ for $X$ and $w_{y}$ for $Y$ ).

$$
f(x, y):-w_{x} / 2<x \leq w_{x} / 2,-w_{y} / 2<y \leq w_{y} / 2
$$

Similarly, they calculated eigenvector of the left $e_{L}$ and right $e_{R}$ footprints then create a covariance matrix $H$. Levenberg-Marquart learning method combined with HMMs (Hidden Markov Model) for both the feet to achieve better performance by Levenberg-Marquart learning method. LM method is a blending of Newton method and gradient descent algorithm. Weight is updated by eq. 10 .

$$
\begin{array}{r}
w(n+1)=w(n)-\left(Z^{T} \cdot Z+\lambda I\right)^{-1} \cdot Z^{T} \mathcal{\varepsilon}(w(n)) \\
(Z)_{n i} \equiv \frac{\partial \varepsilon^{n}}{\partial w_{i}}
\end{array}
$$

where $\varepsilon^{n}$ is the error for $n$th pattern,and $\lambda$ is step size. The recognition rate achieved by HMM blended Levenberg-Marquart learning method is only $64 \%$ with a maximum of $80 \%$ correctly classified samples of 11 subjects.

\subsection{Footprint Heavy Pressure Surface}

Ref. [41], uses Gauss curvature and average curvature on footprint heavy pressure surfaces pick-up to obtain the segmented footprints. The human physical characteristics decide heavy pressure surfaces to realize the identity through abstracting the foot-body pressure images and analyzing their shape features. In preprocessing phase, Gauss function in eq. 11:

$$
h(n)=c \cdot e^{-n^{2} / 2 \sigma^{2}}
$$


filters the image noise. Here normalization coefficient $c$ does not affect the mean of the original image. The samples ranges between $-3 \sigma \leq n \leq 3 \sigma$ used to satisfy eq. 12 .

$$
\sum_{\infty}^{-\infty} h(n)=1
$$

where, $n$ be the slope of integer lies between $-N$ and $N$. Consider $f(u, v)$ be the distance from the dispersed curved surface $u$ to the parametric surface point $v$ in 3D space. Then eq. 13 represents a dispersible parametric surface.

$$
X(u, v)=[u, v, f(u, v)]^{T} \forall u=1,2, . ., m \forall v=1,2, . ., n
$$

If $f_{u}$ and $f_{v}$ be the first order derivative and $f_{u u}, f_{v v}$, and $f_{u v}$ be the second order derivative of $f(u, v)$ then Gauss curvature is obtained as eq. 14, and average curvature is calculated as eq. 15

$$
\begin{gathered}
H=\frac{\left(1+f_{v}^{2}\right) f_{u u}+\left(1+f_{u}^{2}\right) f_{v v}-2 f_{u} f_{v} f_{u v}}{2\left(1+f_{u}^{2}+f_{v}^{2}\right)^{3 / 2}} \\
K=\frac{f_{u u} f_{v v}-f_{u v}^{2}}{\left(1+f_{u}^{2}+f_{v}^{2}\right)^{2}}
\end{gathered}
$$

The Gauss curvature and average curvature represents the principal curvatures of the surface by eq. 16 :

$$
k_{1}=H+\sqrt{H^{2}-K} k_{2}=H-\sqrt{H^{2}-K} \forall k_{1} \geq k_{2}
$$

The pressure surface resembles spherical cap in geometry and has a vital role in the human identification. The geometrical shape index presented in eq. 17 gives an unique value for each footprint.

$$
S_{i}=\frac{2}{\pi} \times \arctan \left(\frac{k_{1}+k_{2}}{k_{1}-k_{2}}\right), \forall S_{1} \epsilon[-1,1]
$$

With 165 samples from 70 subjects, they show the simplicity and robustness of method and extensive application in the criminal investigation, but nothing has been written for matching score.

\subsection{Footprint Similarity for Gait Analysis}

Ref. [54] has introduced a technique to estimate footprint comparison of patient's walking pattern(gait) which is initially unstable and as the treatment goes on it becomes stable to determine the health status. The method initially starts with the edge detection and noise reduction (using the median filter to reduce salt and pepper noise) of 1200 dpi footprint image. Laplace transform has employed for binarization of the footprint. Edge of the binary image used erosion and dilation for smoothening. The distance is given by $d(1 / m \times n p)$ for all $n p$ points from centroid of footprint. Eq. 18 obtains the similarity measure it uses DFT of foot image

$$
d(t)=a_{0}+\sum_{n=1}^{m / 2}\left(a_{n} \cos 2 n \pi t+b_{n} \sin 2 n \pi t\right)
$$

where $m$ is the even number of sampling points, $a_{0}, a_{n}$, and $b_{n}$ are the Fourier coefficients, $t$ is the normalization parameter ranges from $1 / m$ to 1 . The spectrum is calculated as eq. 19 , likewise patient's spectrum is calculated by eq. 20 .

$$
\begin{gathered}
c_{n}=\sqrt{a_{n}^{2}+b_{n}^{2}} ; \forall n=1,2,3, . ., m / 2 \\
\hat{c}_{n}=1,2,3, . ., m / 2
\end{gathered}
$$


The matching spectra between reference and matching footprint presented in eq. 21 gives the similarity of the footprint patterns.

$$
R=1-\frac{1}{2} \sum_{n=0}^{m / 2}\left|c_{n}-\hat{c}_{n}\right|
$$

Inverse DFT removes high-frequency components to produce smoothed edges. The footprint is reconstructed using eq. 22

$$
d(t)=a_{0}+\sum_{n=1}^{k}\left(a_{n} \cos 2 n \pi t+b_{n} \sin 2 n \pi t\right)
$$

The three module of this system includes: (i). Image acquisition achieved by simple flatbed scanners at 600 dpi. (ii). Preprocessing to normalize the image as per requirement and extract the distinct features of footprint image. Canny edge detection with a threshold applied to original footprint image $B$ gives a binarized image $B_{1}$

$$
B_{2}(x, y)=\max \left(\operatorname{bin}_{b}(B)(x, y), B_{1}(x, y)\right)
$$

$B_{2}(x, y)$ in eq. 23 is an image operated to a binary threshold value $b$ using operator $\operatorname{bin}$ on $B$. This binarized image $B_{2}$ is reduced to morphological expansion using a square structuring component $S$ to meet the boundary using eq. 24 :

$$
B_{3}=B_{2} \oplus S=\left\{(x, y) \mid S_{x, y} \cap B_{2} \neq \varnothing\right\}
$$


where $S_{x, y}$ is a shifting of $S$ by coordinate value of $(x, y)$. The binarized image $B_{4}$ is obtained using $B_{3}$ on the removal of small white Binary Large Objects (BLOBs) and a filling of whole black BLOBs except the background. Morphological erosion operation on $B_{4}$ is applied to get $B_{5}$ in eq. 25:

$$
B_{5}=B_{4} \otimes S=\left\{(x, y) \mid S_{x, y} \subseteq B_{4}\right\}
$$

The angle between the $y$ - axis and the major axis $\Theta$ (calculated by eq. 27-29) estimates the best-fitting ellipse for rotational alignment of the matching footprint. Let $B$ is the binary $n \times m$ image with $A$ number of white pixels, then the centre of mass $C(\bar{x}, \bar{y})$ is calculated by eq. 26 (for both ellipse and the binary image $B$ ).

$$
\begin{aligned}
& \bar{x}=\frac{1}{A} \sum_{i=1}^{n} \sum_{j=1}^{m} j B(i, j) \\
& \bar{y}=\frac{1}{A} \sum_{i=1}^{n} \sum_{j=1}^{m} i B(i, j)
\end{aligned}
$$

Let,

$$
\begin{aligned}
x^{\prime} & =x-\bar{x} \\
y^{\prime} & =y-\bar{y}
\end{aligned}
$$

then,

$$
\Theta=\frac{1}{2} \arctan \frac{2 \mu_{1,1}}{\mu_{2,0}-\mu_{0,2}}
$$

where,

$$
\begin{aligned}
& \mu_{1,1}=\sum_{i=1}^{n} \sum_{j=1}^{m}\left(x_{i j}^{\prime}\right)^{2} B(i, j) \\
& \mu_{2,0}=\sum_{i=1}^{n} \sum_{j=1}^{m} x_{i j}^{\prime} y_{i j}^{\prime} B(i, j) \\
& \mu_{0,2}=\sum_{i=1}^{n} \sum_{j=1}^{m}\left(y_{i j}^{\prime}\right)^{2} B(i, j)
\end{aligned}
$$

Three main features namely Eigenfeet using PCA-based approach, minutiae, and shape are utilized in recognition of footprint. and (iii). Matching and Decision module observed a value of FAR as $2.5 \%$, and FRR as $3.13 \%$.

\subsection{Feature Selection and Matching}

Ref. [29] elaborated different features for footprint matching. The silhouette is a feature based on contour distance in two dimensions onto the eigenspace spanned by the 40,100, 200 or 400 most significant eigenvectors of co-variance matrix $C$ to the centroid, length and enclosed area of silhouette polygon it requires dynamic time warp (DTW) matching classifier [73]. Let $s_{k}$ be the contour distances given by eq. 30 .

$$
s_{k}=\left|S_{k}-C\right| \forall k \in\{1, . ., l(S)\}
$$

where $S=\left\{S_{1}, S_{2}, \ldots, S_{l(s)}\right\}$ is the silhouette polygon, and $L(S), A(S)$ are the length and enclosed area to obtain feature vector presented in eq. 31.

$$
f_{1}=\left(s_{1}, s_{2}, \ldots, s_{l(s)}, L(S), A(S)\right)
$$


The $C$ is calculated as in subsection 5.6. The shape of the foot [43] is a feature based on 15 local foot widths and positions, while Eigenfeet [70] is the projection of sub-sampled footprint onto feature space spanned by 20 principal components, both shape, and Eigenfeet features can classify based on Manhattan distance[74]. The feature vector shown in eq. 32:

$$
f_{2}=\left(\omega_{1}, \omega_{2}, . ., \omega_{L}\right)
$$

consists of exactly $L$ components given by eq. 33 for projection onto eigenspace.

$$
\omega_{i}=u_{i}^{T} \Phi
$$

Here $\Phi$ is given by eq. 34

$$
\Phi \sim \sum_{i=1}^{L} \omega_{i} u_{i}
$$

and $u_{i}$ be the set of Eigenfeet $\forall i \in 1,2, . ., L$. After normalizing foot, it is equally divided into $N$ vertical slices $V_{0}, \ldots, V_{N-1}$. Let $\chi$ be the characteristic function and $S_{y}$ be the $y$-monotone polygon to compute the width using eq. 35 of a segment of the foot of size $m \times n$ with $V_{i} \cap S_{y}, \forall i=0,1, . ., N-1$.

$$
w_{i}=\frac{N}{n} \sum_{j=1}^{n} \sum_{k=1}^{m} \chi V_{i} \cap S_{y}(j, k)
$$

Then the feature vector for width is given as eq. 36 with $N=15$.

$$
f_{3}=\left(w_{0}, \ldots, w_{N-1}\right)
$$

A Euclidian distance based classifier classifies sole print [75] which is a variance of 288 overlapping blocks in the edge-detected image; it also classifies toe length which includes five toe lengths and four inter-toe angles. If $\alpha$ is inter-toe angle, then feature vector for toe length can be given by eq. 37.

$$
\begin{gathered}
f_{4}=\left(L_{1}, \alpha_{1}, L_{2}, \alpha_{2}, L_{3}, \alpha_{3}, L_{4}, \alpha_{4}, L_{5}\right) \\
f_{5}=\left(\sigma_{1}^{2}, \sigma_{2}^{2}, . ., \sigma_{288}^{2}\right)
\end{gathered}
$$

The feature vector presented in eq. 38 defines sole print, which consists of extraction of the variance of 288 overlying chunks of size $24 \times 24$ pixels each. NIST's bozorth algorithm can classify minutiae using the mindtct minutiae extractor on ballprint region following the big toe matcher[76]. For each value of $i$ from 1 to $n, f_{i}$ represents the elements of $f$, and for each value of $j$ from 1 to $m, t_{j}$ denotes the reference template for matching. If $e$ in eq. 39 is an evaluation function of Silhouette algorithm[70] then:

$$
e(f, t)=\alpha D(f, t)+\beta\left|f_{n-1}-t_{m-1}\right|+\gamma\left|f_{n}-t_{m}\right|
$$

where $D$ resembles silhouette data sets employing a cost function shown in eq. 40 :

$$
c(i, j)=\left(f_{i}-t_{j}\right)^{2}
$$

on DTW, and the minimum distance both the dataset is given by eq. 41 .

$$
D(f, t)=d(n, m)
$$

If the value of $i=j=1$, then $d(i, j)=0$, for a value of $i>1 ; j=1, d(i, j)=c(i, j)+d(i-1,1)$, similarly for $i=1 ; j>1, d(i, j)=c(i, j)+d(1, j-1)$, or otherwise $d(i, j)=c(i, j)+\min (d(i-$ $1, j), d(i, j-1), d(i-1, j-1))[12]$. 


\subsection{Estimation of Stature from Footprint and Foot Outline Dimensions}

Ref. [30] had applied 2080 footprint and foot outline dimensions collected from 1040 adult male of 18 to 30 years old for ten and eight measurements respectively, for a particular tribe of criminal background which helps in an estimation of the physical build of a criminal (refer Figure 4a and Figure $4 \mathrm{~b}$ to understand the meaning of $\mathrm{T}-1, \mathrm{~T}-2, \ldots, \mathrm{T}-5)$. His study also signifies the utmost importance of footprint into understanding the crime scene investigations. This study indicates statistically important bilateral asymmetry of T-2 and T-5 length in the footprint and T- 1 length, T-4 length, and breadth at the ball in foot outline. These features indicate a close relationship between the stature and these measures with highest positive correlation coefficients of toe length measurements (0.82-0.87). Regression analysis gives better reliability with smaller mean errors $(2.12-3.92 \mathrm{~cm})$ in the estimation of stature $(3.29-4.66 \mathrm{~cm})$. This study motivates to conduct similar kind of research to carried out on other endogamous groups for proving the effect of genetics and environment in forensic terms from different parts of the world.The footprint always has a significant role in crime scene investigation.

Later, Ref. [77] uses a sample of 400 adult Malay participants consisting of 200 males and 200 females using an ink less shoe print kit for investigation of features of the toes, elevations in the toe line, phalange marks, flatfoot condition, pits, cracks, corns and so forth. The occurrence of the fibularis-type foot is the largest, followed by tibialis-type. Then intermediate-type and medullaris-type are determined to be the least frequent in both the sexes which is different sequence observed in north Indian population.

\subsection{Load Distribution Sensor using Fuzzy Logic}

Ref. [56] has collected one step walking foot pressure images from 30 volunteers from 20 to 85 years old using a mat type load distribution sensor of $330 \mathrm{~mm} \times 1760 \mathrm{~mm}$ size comprised of 128 vertical and 64 horizontal electrode sheets with a vertical interval of $7 \mathrm{~mm}$ and a horizontal interval of $5 \mathrm{~mm}$. The fuzzy classifier is defined by eq. 42 .

$$
\mu_{\text {person }}=\sum_{i=0}^{38} \mu_{F, i}\left(f_{i}(X)\right)
$$

where $\mu_{\text {person }}$ is the fuzzy degree of a person, $X$ is a walk data, and $\mu_{F, i}$ is the total fuzzy degree, has applied to all the obtained dynamic feature weight movement and footprint feature foot shape during walking. The fuzzy classification of a person has inferred from out of six samples per volunteer five samples used for training and one sample for test data. This method gives $6.1 \%$ EER and $13.9 \%$ FRR in verification ( $1: 1$ collation) and identification ( $1: N$ collation).

\subsection{Foot Pressure Change using Neural Network}

Ref. [58] obtain the center of pressure (COP) features (position and the movement) for a personal identification system captured from 11 volunteers (10 samples/volunteer) standing statically on the load distribution sensor with a slipper. The load distribution sensor Nippon Ceramic Co., Ltd., NCK is composed of 64 horizontal $\times 128$ vertical electrodes sheets. The size of each such sheet is $330 \mathrm{~mm}$ $\times 640 \mathrm{~mm}$, and the interval between two sheets is $5 \mathrm{~mm}$. The inclination angle of the slipper is $9.5^{\circ}$. This system has extracted 16 features. Out of 10 samples of the dataset, five rows has used as the test set, and remaining five has used for comparison. This system has developed with a k-out-of-n system and a neural network model under less information, time and small space and experimented input test data to both the systems. The correlation coefficients of the k-out-of-n method for matching has calculated as eq. 43 .

$$
r=\frac{\sum_{i=1}^{n}\left(x_{i}-\bar{x}\right)\left(y_{i}-\bar{y}\right)}{\sqrt{\sum_{i=1}^{n}\left(x_{i}-\bar{x}\right)^{2}\left(y_{i}-\bar{y}\right)^{2}}}
$$


with $x_{i}$ be the test data in $t_{i}, y_{i}$ denotes the template in $t_{i}, \bar{x}$ and $\bar{y}$ express the average of the test and template data respectively. The Neural Networks based technique for recognition consist of 16 input neurons (number of features), 17 hidden neurons, and 11 output neurons (number of users). These methods show FRR of $28.6 \%$ and FAR of $1.3 \%$ for the k-out-of-n system and FRR of $12.0 \%$ FAR of $1.0 \%$ in neural network.

\subsection{Wavelet and Fuzzy Neural Network}

Ref. [31], gives a method based on Wavelet and Fuzzy Neural Network with a claimed recognition rate of $92.8 \%$. Wavelet distinguishes the edge of the footprint to identify the statistical features of the four different shapes (triangle, ellipse, circular and irregular) of toe images such as angle, length, and area. The feature vector given in eq. 44 :

$$
V=\left(v_{1}=E, v_{2}=r_{c}-r_{i} / r_{i}, v_{3}=S, v_{4}=A C, v_{5}=\alpha_{1}, v_{6}=\alpha_{2}\right)
$$

is constituted by six parameters, where $E=p / q$ is the eccentricity of toe region, $v_{2}$ calculates the ratio of the radius difference between circumscribed circle and inscribe circle to the radius of the inscribed circle, $S=r_{i} / r_{c}$ is the sphericity of toe region, $A C$ calculates the area of toe region, $v_{5}$, and $v_{6}$ represents the angular parameters of the toe. This technique uses six-six membership functions of ellipsoidal toe shape, triangular toe shape, circular toe shape, and irregular toe shape. The singular judgment factor obtained from these membership functions are operated by AND to get the comprehensive judgment vector. The neural network has four input, and four output neurons for each shape types. Comprehensive judgment vector is compared with four model vectors to determine the distance vector to feed into neural networks to decide. A sum of 80 samples extracts 320 toe images for different shapes.

\subsection{Modified Sequential Haar Energy Transform (MSHET)}

Ref. [32] introduce modified sequential haar energy transform technique. The human footprint is robust as it does not change much over the time, universal, easy to capture as it does not need specialized acquisition hardware. Initially, the algorithm starts with capturing the left leg images acquired in different angles without provisioning any particular lighting in this setup from 400 subjects. The obtained images are then normalized for the key points and cropped respectively and converted to grayscale from RGB. Feature extraction methods Linear Predictive Coding (LPC), and Linear Predictive Cepstral Coefficients (LPCC) given by [78] has applied to the obtained images to enhanced it further by transforming to a matrix format separated into odd and even matrices of $256 \times 256$ pixels. Sequential Modified Haar $(\mathrm{SMH})$ transforms employed to the resized images $(4 \times 4$ blocks) to retrieve a Modified Haar Energy (MHE) features determined by eq. 45:

$$
M H E_{i, j, k}=\sum_{p=1}^{4} \sum_{q=1}^{4}\left(C_{p, q}\right)^{2}
$$

for level of decomposition $i$, neighborhood detail $j$ is, and block number $k$ from 1 to 16 . The SMH wavelet can map integer-valued signals onto integer-valued signals yielding the property of perfect restoration. The Euclidean distance compares MHE feature with the feature vectors stored in the database and yields $92.375 \%$ percent of accuracy.

\subsection{Multibiometrics Rank-Level Fusion}

Ref. [79]reviews an approach for the personal recognition employing a nonlinear rank-level fusion of multiple biometrics representations. This approach gives a direction to apply rank-level fusion in the footprint biometrics. It compares results from openly available multi-biometrics (NIST BSSR1 database from 517 subjects) scores and real palm print biometrics data to determine the rank-level 
fusion employing Borda count, logistic regression / weighted Borda count, highest rank system, and Bucklin system. The empirical results presented suggest that significant performance improvement on the recognition accuracy from $94 \%$ to $99 \%$. The precise experimental results also indicate that the nonlinear rank-level approach excels over other rank-level fusion methods.

\subsection{Dynamic Plantar Pressure}

Ref. [61] has used a sum of 1040 dynamic foot pressure patterns which are usable immediately and collected from 10 volunteers (104 samples per subject) based on automatic spatial alignment for achieving high classification rate (CR) of $99.6 \%$ regardless of automatic dimensionality reduction. These patterns reveal the expeditions of all body parts are different leads to a conclusion that pointedly indicates inter-subject pressure pattern uniqueness. The pre-processing requirement is also minimal, and as they might collect while uninterrupted gait using in-floor systems, foot pressure-based identification resembles to have the broad perspective in the security and health industries.

\subsection{Discrete correlation of Footprint Image}

According to the survey presented by Ref. $[33,80]$, the last decade was devoted for footprint based feature extraction for personal recognition. They have discussed and concluded some most prominent feature extraction techniques. The algorithms are commonly divided into three major stages (i) image pre-processing-is the method used to diminish the noise, (ii) feature extraction from images such as Center of Foot Pressure (COP), geometric constraint, toe shape, triple feature, vibration feature, tactile sensor feature, image feature, walking pattern, stepping pattern, footprint arch, location and weight and (iii) template matching to improve the performance by enhancing the matching score. The authentication based on footprint owns sufficient security without any shortcoming of relying on sensitive data required by high-security applications.

\subsection{Orientation Feature-Newborn Baby}

Ref. [81] has developed an online footprint based system for identification of newborn and infant, which is a critical issue for the places where multiple births occur. This approach captured high-quality images of size $691 \times 518$ as compared to traditional offline methods. The offline footprint images go obscured due to: (i) Use of incompetent stuff like ink pads, paper, cylinder and so forth; (ii) Lack of training of personnel for footprint image acquisition; (ii) Oily matter on baby's skin gives non-recognisable prints; (iv) The epidermis is quickly distorting the ridges upon touch and filling the valleys within ridges with ink due to less thickness; (v) Tiny size of the infant's ridges. The preprocessing phase changes the orientation and scale to normalize the footprint image. Initially, they start with image segmentation to extract footprint $G(x, y)$ with threshold $T$ to get segmented binary image. Next phase calculates the centre of footprint $C(x, y)$ in binary image $B(x, y)$ by using eq. 46 :

$$
C(x, y)=\text { centre }\left\{\frac{\sum_{x} x \sum_{y} B(x, y)}{\sum_{x, y} B(x, y)}, \frac{\sum_{y} y \sum_{x} B(x, y)}{\sum_{x, y} B(x, y)}\right\}
$$

The obtained image is then normalized to $C(x, y)$ and a region of interest (ROI) is cropped. The database consists of 1938 images from 101 newborns applied in recognition stage on four orientation feature-based methods, Ordinal Code, BOCV, Competitive Code, and Robust Line Orientation Code. The highest recognition rate achieved has $96 \%$.

\subsection{Manifold Feature Extraction using PCA and ICA}

Ref. [35,36,82,83] gives a method based on manifold feature extraction. Ref. [37] has extracted fingerprint minutiae feature and PCA footprint features of newborn on Raspberry Pi system. Starting from capturing the footprint, preprocessing provides binary images; these binary images then yield COP data; subsequently, feature extraction phase extracts features; PCA trains the data, and stochastic 
415

416

gradient descent algorithms have utilized to match the footprint for recognition. PCA can do recognition with SVM, or Wavelet-based Fuzzy Neural Network can also be used for recognition. The feature has extracted based on Gabor filter and Discrete Wavelet Transforms (DWT). The maximum recognition rate obtained has $90.35 \%$.

Ref. [84] has used multiple features to automated identification based on segmentation. Figure 6 exhibits different parts of the footprint to estimate the statistical details. The system is further extended to identify the human by footprints using ICA and PCA. This system covers various statistical computations of the footprint indices for the identification. A hundred-time fraction of minimum foot width and overall foot width gives the Instep-Foot Index. Ball-Foot Index can have calculated as a hundred-time fraction of maximum foot width and overall foot width. A hundred-time portion of heel width and heel length yields Heel-Index. Toe-Index is calculated as a hundred-time fraction of the toe width and toe length. Likewise, a hundred-time fraction of maximum foot width and heel width estimates Ball Heel Index. Finally, a hundred-time portion of the toe width and maximum foot width determines the Toe Ball Index.

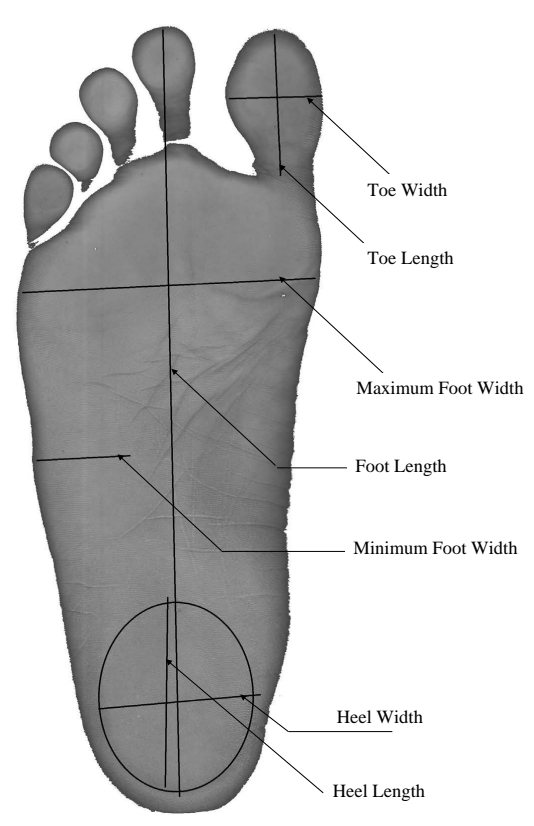

Figure 6. Diffrent parts of the footprint to estimate statistical details.

Ref. [38] exhibit the use of PCA and Independent Component Analysis (ICA) for footprint recognition. PCA initially computes the covariance matrix using eq. 47 :

$$
C=(X-\bar{x}) \times(X-\bar{x})^{T}
$$

$\forall$ mean $\bar{x}$ in dataset $X$. The relationship between eigenvectors $V$ and $C$ in $V^{-1} \times C \times V=D$ gives eigenvalues $D . V$ is stored in descending order of $D$ to evaluate the projected data $\wp$ in $V$-dimensional vector space by relation eq. 48 :

$$
\wp=\left[V^{T}(X-\bar{x})^{T}\right]^{T}
$$

The computation in ICA begin with centering to get centered observation vector of $x$ as shown in eq. 49.

$$
x_{c}=x-\bar{x}
$$


After getting unmixing matrix using the centralized data the actual estimates of the independent components can be obtained by eq. 50 .

$$
\hat{s}=A^{-1}\left(x_{c}+\bar{x}\right)
$$

The observation vector $x$ is now transformed to whitening vector $x w$ and satisfies the covariance matrix $E\left\{x_{w} x_{x}^{T}\right\}$ of $x_{w}$ to unity.

$$
E\left\{x x^{T}\right\}=V D V^{T}
$$

Eq. 51 presents the breakdown of the covariance matrix of $x$. Further, eq. 52 gives whitening transform of $x_{w}$.

$$
x_{w}=V D^{-1 / 2} V^{T} x
$$

Also, Whitening converts the mixing matrix into a new orthogonal form by eq. 53

$$
\begin{array}{r}
x_{w}=V D^{-1 / 2} V^{T} A s=A_{w} S \\
\text { hence, } E\left\{x x^{T}\right\}=A_{w} E\left\{s s^{T}\right\} A_{w}^{T}=I
\end{array}
$$

Therefore, whitening reduces the number of components to be estimated. For 21 subjects [38] claims ICA with the RR of $97.23 \%$ and PCA $95.24 \%$ RR.

\subsection{Foot-boundary for Crime Scene Investigation}

Later, Ref. [85] gives a computational analytics of crime scene investigation, which is a meeting point of science, logic, and law using foot boundary detection. Mostly, in the manual crime scene, the investigator investigates based on blood spots, hair, fingerprints, and available piece of belongings

\begin{tabular}{|c|c|c|c|}
\hline Authors & Main idea & Gap & $\mathbf{R R}$ \\
\hline Nakajima et al. [43] & $\begin{array}{l}\text { Euclidean distance based person identification among } 10 \text { men } \\
\text { using normalized static footprint }\end{array}$ & $\begin{array}{l}\text { user should make stand up posture every time; recognition rate } \\
\text { is insufficient for practical uses; only } 10 \text { subjects. }\end{array}$ & $85 \%$ \\
\hline Collins et al. [51] & $\begin{array}{l}\text { Viewpoint based template matching of body silhouettes captures } \\
\text { height, width, and part proportions of the human body }\end{array}$ & $\begin{array}{l}\text { MIT database } 25 \text { subjects exercising on a treadmill, the } \\
\text { U.Maryland database with } 55 \text { individuals, and the University of } \\
\text { Southampton database } 28 \text { subiects walking indoors on track }\end{array}$ & $76 \%$ to $100 \%$ \\
\hline Jung et al. $[40,68,69]$ & $\begin{array}{l}\text { Unconstrained person recognition method using dynamic } \\
\text { footprint in which person identification is done using } \\
\text { position-based quantization of COP from the shoe-type pressure } \\
\text { sensor and Hidden Markov Model blended Levenberg-Marquart } \\
\text { learning. }\end{array}$ & $\begin{array}{l}\text { highly correlated data as the images were collected in a day; } \\
\text { using shoe-type sensor could be a significant constraint in the } \\
\text { view of users;only } 11 \text { subjects. }\end{array}$ & $64 \%$ to $80 \%$ \\
\hline Kale et al.[52] & $\begin{array}{l}\text { Invariant gait recognition approach; incorporated views for gait } \\
\text { recognition at any arbitrary angle to the camera on a perspective } \\
\text { plane }\end{array}$ & $\begin{array}{l}12 \text { subjects, walking onward straight lines at varying } \\
\text { values of azimuth angle } \theta \text { from } 0^{\circ}, 15^{\circ}, 30^{\circ}, 45^{\circ} \text { and to } 60^{\circ} \\
\left(G_{0}, G_{15}, G_{30}, G_{45} \text {, and } G_{60}\right)\end{array}$ & Not stated \\
\hline Wang et al. & $\begin{array}{l}\text { Put forward a kind of footprint heavy pressure surfaces pick-up } \\
\text { design based on Gauss curvature and average curvature. This } \\
\text { process can pick-up effectively footprint heavy pressure surfaces } \\
\text { that are the area of interest. }\end{array}$ & $\begin{array}{l}\text { filtered images may have artificial images. } 70 \text { subjects and } 165 \\
\text { samples. }\end{array}$ & Not stated \\
\hline Boulgouris et al. [53] & HMM is applied in gait stances & $\begin{array}{l}71 \text { subjects; the right subject gives high confidence in top } 10 \\
\text { matches. Gait alone is not sufficient to provide reliable and } \\
\text { accurate recognition; this gives clear implication to use gait in } \\
\text { conjunction with other biometrics traits for best performance in } \\
\text { personal identification. }\end{array}$ & upto $86 \%$ \\
\hline Makihara et al. [62] & $\begin{array}{l}\text { View transformation model in the frequency domain features to } \\
\text { generate the training set of multiple subjects from features of one } \\
\text { or a few of the view directions }\end{array}$ & $\begin{array}{l}20 \text { subjects for gait recognition from } 24 \text { perspective directions at } \\
\text { every } 15 \text { degrees }\end{array}$ & $\begin{array}{l}\text { verification rate at } \\
\text { a FRR is } 10 \%\end{array}$ \\
\hline Kuragano et al. [54] & $\begin{array}{l}\text { estimate footprint comparison of patient's walking pattern(gait) } \\
\text { which is initially unstable and as the treatment goes on it } \\
\text { becomes stable to determine the health status }\end{array}$ & $\begin{array}{l}\text { not used for personal identification rather it is used to check } \\
\text { the rehabilitation status of any person using the footprint and } \\
\text { walking pattern }\end{array}$ & NA \\
\hline Hewson et al.[55] & $\begin{array}{l}\text { gait detection and a balanced signature extracted from a force } \\
\text { plate to fall prevention in old aged person in the PARAchute } \\
\text { project }\end{array}$ & $\begin{array}{l}\text { The obtained gait sequence enables the calculation of kinematic } \\
\text { and spatiotemporal variables already identified as appropriate in } \\
\text { an examination of the risk of falling }\end{array}$ & NA \\
\hline Yun et al. [42] & $\begin{array}{l}\text { Neural Network based Biometric User Identification with } \\
\text { Dynamic Footprint. Recognizes the user with a well-trained } \\
\text { neural network. }\end{array}$ & $\begin{array}{l}10 \text { subjects while walking over the UbiFloorII; numerous input } \\
\text { nodes in the neural network, causing heavy computation loads; } \\
10 \text { subjects. }\end{array}$ & $92 \%$ to $96 \%$ \\
\hline Uhl and Wild [27-29] & $\begin{array}{l}\text { Personal identification using Eigenfeet, Ballprint and Foot } \\
\text { geometry biometrics focusing on characteristics such as the foot } \\
\text { widths, to improve recognition rates }\end{array}$ & only 16 subjects. & $97 \%$ \\
\hline
\end{tabular}
makes the task very long and slow. Foot biometry has largely ignored so far. This approach takes the high-definition pictures of the available footprint of any form. These images then used for identification purpose based on foot boundary.

Table 1. Review and gap analysis of existing methods. 


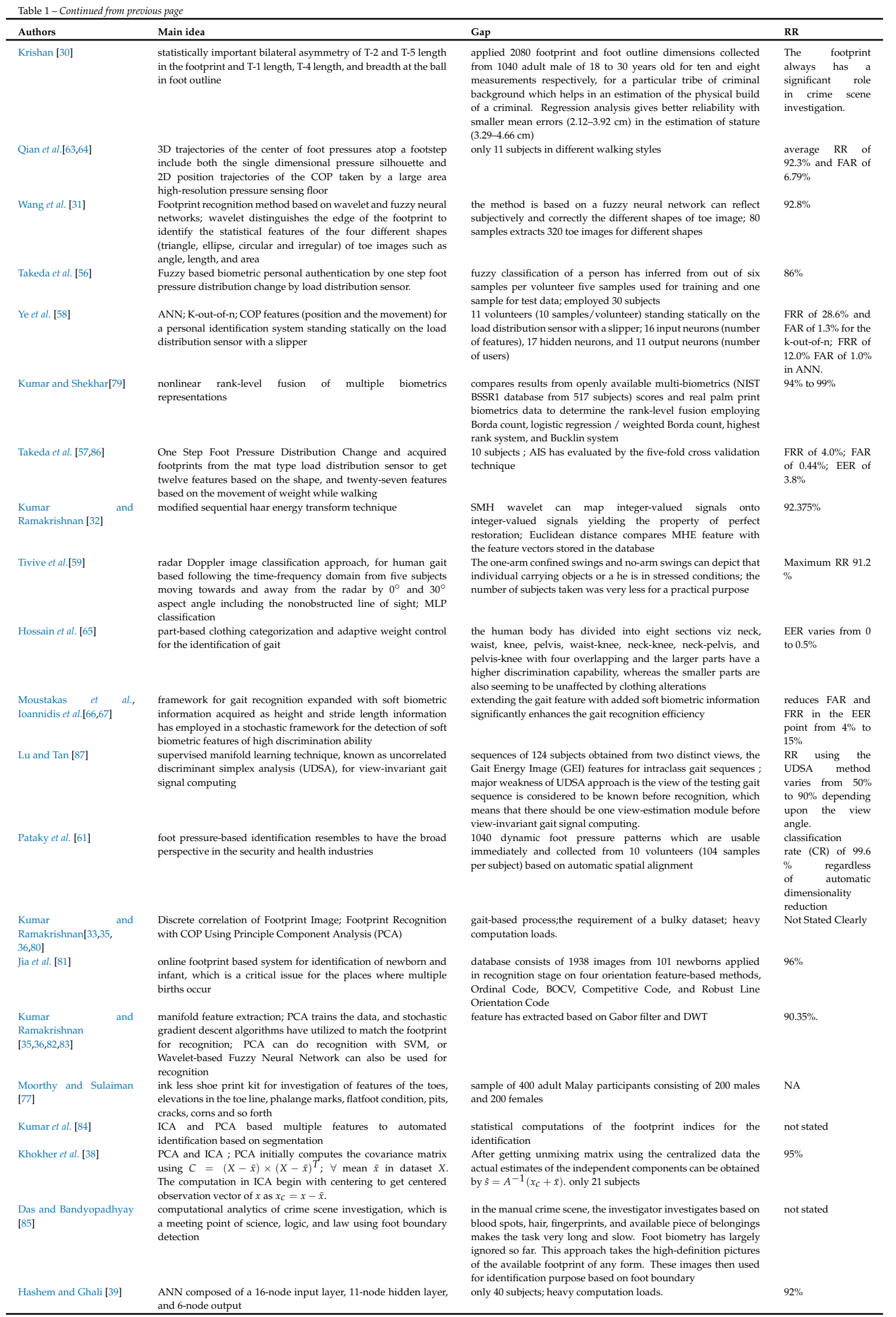




\subsection{ANN Foot Features}

Ref. [39] have extracted sixteen geometric foot features of human footprints collected from 40 subjects. The ANN composed of a 16-node input layer, 11-node hidden layer, and 6-node output later to recognize 40 subjects for a testing : training set of 40:120 images. They have used ANN for the the feature extraction and recognition with a highest recognition rate of $92 \%$.

\section{Discussion}

Ref. [20] had analysed the uniqueness of human footprints. Footprint (and foot) morphology is significant since it explains the unique character of each person's footprints[43]. Hospitals used footprints to identify an infant by its footprints while Austria utilized its legal capabilities [23,88]. Since footprints are not intended to support large-scale high-security applications, such as electronic banking, the storage of features does not necessarily imply security threats. A person without having hands can use footprint biometrics in the legal capacity for identification. On the contrary, due to the practice of wearing shoes, it is difficult for imposters to obtain footprints for forgery attacks. Thus, footprint-based recognition may correspondingly be the best substitute for highest-security applications this motivates to develop an access control system based on footprint expected to have the highest recognition rate without the need for additional hardware.

Table 1 covers latest review summary for diffrent footprint based biometric identification. Ref. [40, $68,69,89]$ developed a technique to calculate COP(Centre of Pressure) based on Hidden Markov Model. Ref. [36]encompasses a shoe-type pressure sensor and HMM(Hidden Markov Model). A comprehensive evaluation model comes into existence for establishing to recognize the toe shape using the neural network and fuzzy logic by Ref. [90]. Self-organizing map [91] for automation of the process, ART2 [92] for optimization of footprint recognition, a trace transform technique for parallel line [93], UbiFloor2 [42] using neural network, principle component analysis (PCA) [36], wavelet transform [32,94] based footprint recognition; these are some methods applied during the last decade. Multimodal biometric is another method of person identification[27,95,96]. [97] introduces a different classification of biometric. According to [29], identify target application domains for footprint-based authentication. Ref. [38,98] put forward texture and shape oriented footprint features using PCA and Independent Component Analysis (ICA). Artificial Neural Network (ANN) based work for footprint feature extraction, and pattern recognition has been carried out to get a recognition rate of 92.5\% [39].

\section{Conclusion}

Two different ways of the foot for identification have discussed here. The first method based on behavior or gait and second purely based on the human footprint. The gait based method takes a significant amount of data per person to recognize. One can recognize the thought, whether it is positive or negative, of a person while walking. The gait significantly gives the status of a patient, whether recovering or not. It also helps in determining the crime scene and creates the possible theories. The bulk of images slows down the processing capabilities. It is, therefore, not possible to keep gait record of every individual. Most of the researchers took gait as a patient-care method, not a personal recognition method. Through the comprehensive literature survey, the gait based approach is not suitable for personal identification. On the other hand, the footprint-based process requires very few samples per person. Both types of footprint features (morphology, statistical) helps to identify a person. This method applies to high-security zones, such as the airport, crowded places prone to terrorist attack, silicon chip manufacturing agency, nanotechnology research laboratories, public wellness zones. The footprint-based method also plays a significant role in crime scene identification and creation of theories. This technique requires the barefoot person to capture the footprint. With the bulk of data, the recognition rate is higher as similar to fingerprint-based technique. The present study also explained legal capability of the human footprint. 
Acknowledgments: The authors would like thanks to Dr. S. P. Dubey from Rungta College of Engineering and Technology, Bhilai, India, who provided insight and expertise that greatly assisted the research, although they may not agree with all the interpretations/conclusions from this paper. The authors are also immensely grateful to our management Dr. Sourabh Rungta and Mr. Sonal Rungta for their kind support.

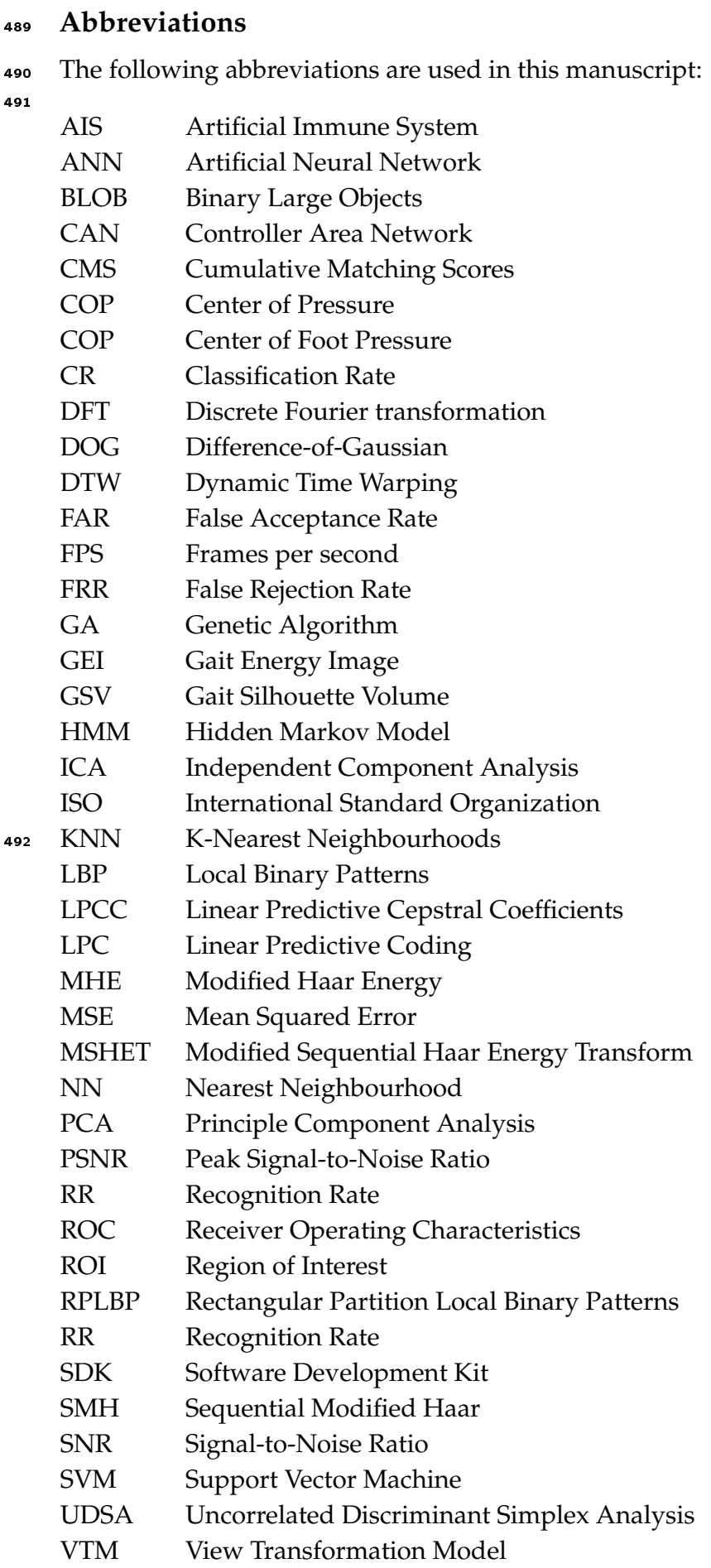


14, 566-567, [http://rstl.royalsocietypublishing.org/content/14/155-166/566.full.pdf+html]. doi:10.1098/rstl.1684.0028.

2. Purkyne, J.E. Commentatio de examine physiologico organi visus et systematis cutanei: Quam pro loco in gratioso medicorum ordine rite obtinendo die XXII. decembris MDCCCXXIII, H.X.L.C. Master's thesis, Vratislaviae : Typus Universitatis, University of Breslau, 1823. The National Library of Medicine believes this item to be in the public domain.

3. Bolle, R.; Pankanti, S. Biometrics, Personal Identification in Networked Society: Personal Identification in Networked Society; Kluwer Academic Publishers: Norwell, MA, USA, 1998.

4. Jain, A.; Hong, L.; Pankanti, S. Biometric Identification. Commun. ACM 2000, 43, 90-98. doi:10.1145/328236.328110.

5. Franke, K.; Ruiz-del Solar, J., Soft-Biometrics: Soft-Computing Technologies for Biometric-Applications. In Advances in Soft Computing - AFSS 2002: 2002 AFSS International Conference on Fuzzy Systems Calcutta, India, February 3-6, 2002 Proceedings; Springer Berlin Heidelberg: Berlin, Heidelberg, 2002; pp. 171-177. doi:10.1007/3-540-45631-7_24.

6. Jain, A.K.; Pankanti, S.; Prabhakar, S.; Hong, L.; Ross, A. Biometrics: a grand challenge. Proceedings of the 17th International Conference on Pattern Recognition, 2004. ICPR 2004., 2004, Vol. 2, pp. $935-942$. doi:10.1109/ICPR.2004.1334413.

7. Jain, A.K.; Ross, A.; Prabhakar, S. An introduction to biometric recognition. IEEE Transactions on Circuits and Systems for Video Technology 2004, 14, 4-20. doi:10.1109/TCSVT.2003.818349.

. Henry, E. Classification and Uses of Finger Prints; Making of modern law, George Routledge and Sons, 1900.

9. Bolle, R.M.; Connell, J.H.; Pankanti, S.; Ratha, N.K.; Senior, A.W. Guide to Biometrics; Springer, 2004.

10. Farelo, A. A history of fingerprints. Technical report, Fingerprint Examiner at Forensic Support \& Technical Databases, Operational Police Support Directorate , ICPO- INTERPOL, General Secretariat with the precious collaboration of Annalise Wrzeczycki, Lyon, France, 2009.

11. Hennings-Yeomans, P.H.; Kumar, B.V.K.V.; Savvides, M. Palmprint Classification Using Multiple Advanced Correlation Filters and Palm-Specific Segmentation. IEEE Transactions on Information Forensics and Security 2007, 2, 613-622. doi:10.1109/TIFS.2007.902039.

12. Ross, A.; Jain, A. Information Fusion in Biometrics. Pattern Recogn. Lett. 2003, 24, $2115-2125$. doi:10.1016/S0167-8655(03)00079-5.

13. You, J.; Kong, W.K.; Zhang, D.; Cheung, K.H. On hierarchical palmprint coding with multiple features for personal identification in large databases. IEEE Transactions on Circuits and Systems for Video Technology 2004, 14, 234-243. doi:10.1109/TCSVT.2003.821978.

14. Zhang, D.; Lu, G.; Li, W.; Zhang, L.; Luo, N. Palmprint Recognition Using 3-D Information. IEEE Transactions on Systems, Man, and Cybernetics, Part C (Applications and Reviews) 2009, 39, 505-519. doi:10.1109/TSMCC.2009.2020790.

15. Simpson, L.; Dozier, G.; Adams, J.; Woodard, D.L.; Miller, P.; Bryant, K.; Glenn, G. Genetic amp; Evolutionary Type II feature extraction for periocular-based biometric recognition. IEEE Congress on Evolutionary Computation, 2010, pp. 1-4. doi:10.1109/CEC.2010.5585948.

16. Kumar, A.; Zhang, D. Personal recognition using hand shape and texture. IEEE Transactions on Image Processing 2006, 15, 2454-2461. doi:10.1109/TIP.2006.875214.

17. Yoruk, E.; Konukoglu, E.; Sankur, B.; Darbon, J. Shape-based hand recognition. IEEE Transactions on Image Processing 2006, 15, 1803-1815. doi:10.1109/TIP.2006.873439.

18. Kanchan, T.; Krishan, K.; Prusty, D.; Machado, M. Heel-Ball index: An analysis of footprint dimensions for determination of sex. Egyptian Journal of Forensic Sciences 2014, 4, 29 - 33. doi:https:/ /doi.org/10.1016/j.ejfs.2014.02.002.

19. Khan, H.B.M.A.; Moorthy, T.N. Stature estimation from the anthropometric measurements of footprints among Melanaus: an indigenous population of Malaysian Borneo. Canadian Society of Forensic Science Journal 2015, 48, 68-84, [http:/ / dx.doi.org/10.1080/00085030.2015.1019225]. doi:10.1080/00085030.2015.1019225.

20. Robbins, L. The Individuality of Human Footprints. Journal of Forensic Sciences 1978, 23, 778-785. doi:10.1520/JFS10737J.

21. Vernon, W. Forensic podiatry: A review. Axis 2009, 1, 60-70.

22. Krishan, K.; Kanchan, T.; Passi, N.; DiMaggio, J.A. Sexual dimorphism in foot length ratios among North Indian adolescents. Journal of forensic and legal medicine 2015, 36, 96-101. doi:10.1016/j.jflm.2015.09.007. 
23. Nagwanshi, K.K.; Dubey, S. Biometric Authentication using Human Footprint. International Journal of Applied Information Systems 2012, 3, 1-6. Published by Foundation of Computer Science, New York, USA, doi:10.5120/ijais12-450568.

24. Nagwanshi, K.K.; Dubey, S. Design of Enhanced Matcher for Biometric Footprint Recognition. COMPUTER ENGINEERING AND INFORMATION TECHNOLOGY (VOL. 2)-Chapter 18: 3rd International Conference on "Recent Trends in Engineering \& Technology". Elsevier Science and Technology, 2014, Vol. 2, pp. 601-608. 25. Nagwanshi, K.K.; Dubey, S. Statistical Feature Analysis of Human Footprint for Personal Identification Using BigML and IBM Watson Analytics. Arabian Journal for Science and Engineering 2017. doi:10.1007/s13369-017-2711-z.

26. Naples, V.L.; Miller, J.S. Making tracks: The forensic analysis of footprints and footwear impressions. The Anatomical Record Part B: The New Anatomist 2004, 279B, 9-15. doi:10.1002/ar.b.20025.

27. Uhl, A.; Wild, P. Personal identification using Eigenfeet, Ballprint and Foot geometry biometrics. 2007 First IEEE International Conference on Biometrics: Theory, Applications, and Systems, 2007, pp. 1-6. doi:10.1109/BTAS.2007.4401924.

28. Uhl, A.; Wild, P. Footprint-based biometric verification. Journal of Electronic Imaging 2008, 17, 011016-011016-10. doi:10.1117/1.2892674.

29. Wild, P. Single-sensor hand and footprint-based multimodal biometric recognition. Thesis, Naturwissenschaftlichen Fakultät der Universität Salzburg, Salzburg, 2008.

30. Krishan, K. Estimation of stature from footprint and foot outline dimensions in Gujjars of North India. Forensic Science International 2008, 175, 93 - 101. doi:http:/ / dx.doi.org/10.1016/j.forsciint.2007.05.014.

31. Wang, R.; Hong, W.; Yang, N. The Research on Footprint Recognition Method Based on Wavelet and Fuzzy Neural Network. 2009 Ninth International Conference on Hybrid Intelligent Systems, 2009, Vol. 3, pp. 428-432. doi:10.1109/HIS.2009.300.

32. Kumar, V.D.A.; Ramakrishnan, M. Footprint recognition using modified sequential haar energy transform (MSHET). Intl. Journal of Computer science Issuse 2010, 7.

33. Kumar, V.D.A.; Ramakrishnan, M. Legacy of Footprints Recognition- A Review. International Journal of Computer Applications 2011, 35, 9-16.

34. Kumar, V.D.A.; Ramakrishnan, M., Footprint Based Recognition System. In Information Technology and Mobile Communication: International Conference, AIM 2011, Nagpur, Maharashtra, India, April 21-22, 2011. Proceedings; Springer Berlin Heidelberg: Berlin, Heidelberg, 2011; pp. $358-367$. doi:10.1007/978-3-642-20573-6_63.

35. Kumar, V.D.A.; Ramakrishnan, M. A Comparative Study of Fuzzy Evolutionary Techniques for Footprint Recognition and Performance Improvement Using Wavelet-based Fuzzy Neural Network. Int. J. Comput. Appl. Technol. 2013, 48, 95-105. doi:10.1504/IJCAT.2013.056016.

36. Kumar, V.D.A. Footprint Recognition with COP Using Principle Component Analysis (PCA). Journal of Computational Information Systems 2012, 8, 4939-4950.

37. Sivaranjani, S.; Sumathi, S. Implementation of fingerprint and newborn footprint feature extraction on Raspberry Pi. 2015 International Conference on Innovations in Information, Embedded and Communication Systems (ICIIECS), 2015, pp. 1-6. doi:10.1109/ICIIECS.2015.7193087.

38. Khokher, R.; Singh, R.C.; Kumar, R. Footprint Recognition with Principal Component Analysis and Independent Component Analysis. Macromolecular Symposia 2015, 347, 16-26. doi:10.1002/masy.201400045.

39. Hashem, K.M.; Ghali, F. Human Identification Using Foot Features. Intl. J. of Engineering and Manufacturing 2016, 6, 22-31. doi:10.5815/ijem.2016.04.03.

40. Jung, J.W.; Park, K.H.; Bien, Z., Unconstrained Person Recognition Method using Dynamic Footprint; The Institute of Electronics Engineers of KOREA, 2002; Vol. 25, pp. 91-94.

41. Wang, W.; Ping, X.; Ding, Y. Footprint heavy pressure surface pick-up and description. Image and Graphics (ICIG'04), Third International Conference on, 2004, pp. 278-281. doi:10.1109/ICIG.2004.69.

42. Yun, J.; Abowd, G.; Woo, W.; Ryu, J. Biometric User Identification with Dynamic Footprint. 2007 Second International Conference on Bio-Inspired Computing: Theories and Applications, 2007, pp. 225-230. doi:10.1109/BICTA.2007.4806456.

43. Nakajima, K.; Mizukami, Y.; Tanaka, K.; Tamura, T. Footprint-based personal recognition. IEEE Transactions on Biomedical Engineering 2000, 47, 1534-1537. doi:10.1109/10.880106. 
44. Birch, I.; Raymond, L.; Christou, A.; Fernando, M.A.; Harrison, N.; Paul, F. The identification of individuals by observational gait analysis using closed circuit television footage. Science and Justice 2013, 53, 339-342.

45. Hamilton, J. Scottish Murders: From Burke and Hare to Peter Tobin. Glasgow: Waverley Books. Accessed January 2013, 1, 2016.

46. Nirenberg, M. Gait, Footprints, and Footwear: How Forensic Podiatry Can Identify Criminals, 2017.

47. Nirenberg, M. Forensic methods and the podiatric physician. Journal of the American Podiatric Medical Association 1989, 79, 247-252. doi:10.7547/87507315-79-5-247.

48. Nirenberg, M. Meeting a Forensic Podiatry Admissibility Challenge: A Daubert Case Study. Journal of Forensic Sciences 2016, 61, 833-841. doi:10.1111/1556-4029.13037.

49. DiMaggio, J.A.; Vernon, W. Forensic podiatry: principles and methods; Humana Press: Berlin, Heidelberg, 2011. doi:10.1007/978-1-61737-976-5.

50. Baker, R. The history of gait analysis before the advent of modern computers. Gait and Posture 2007, 26, 331-342.

51. Collins, R.T.; Gross, R.; Shi, J. Silhouette-based human identification from body shape and gait. Proceedings of Fifth IEEE International Conference on Automatic Face Gesture Recognition, 2002, pp. 366-371. doi:10.1109/AFGR.2002.1004181.

52. Kale, A.; Chowdhury, A.K.R.; Chellappa, R. Towards a view invariant gait recognition algorithm. Proceedings of the IEEE Conference on Advanced Video and Signal Based Surveillance, 2003., 2003, pp. 143-150. doi:10.1109/AVSS.2003.1217914.

53. Boulgouris, N.V.; Hatzinakos, D.; Plataniotis, K.N. Gait recognition: a challenging signal processing technology for biometric identification. IEEE Signal Processing Magazine 2005, 22, 78-90. doi:10.1109/MSP.2005.1550191.

54. Kuragano, T.; Yamaguchi, A.; Furukawa, S. A Method to Measure Foot Print Similarity for Gait Analysis. International Conference on Computational Intelligence for Modelling, Control and Automation and International Conference on Intelligent Agents, Web Technologies and Internet Commerce (CIMCA-IAWTIC'06), 2005, Vol. 2, pp. 816-822. doi:10.1109/CIMCA.2005.1631569.

55. Hewson, D.J.; Duchêne, J.; Charpillet, F.; Saboune, J.; Michel-Pellegrino, V.; Amoud, H.; Doussot, M.; Paysant, J.; Boyer, A.; Hogrel, J.Y. The PARAChute Project: Remote Monitoring of Posture and Gait for Fall Prevention. EURASIP J. Appl. Signal Process. 2007, 2007, 109-123. doi:10.1155/2007/27421.

56. Takeda, T.; Taniguchi, K.; Asari, K.; Kuramoto, K.; Kobashi, S.; Hata, Y. Biometric personal authentication by one step foot pressure distribution change by load distribution sensor. 2009 IEEE International Conference on Fuzzy Systems, 2009, pp. 906-910. doi:10.1109/FUZZY.2009.5277149.

57. Takeda, T.; Taniguchi, K.; Asari, K.; Kuramoto, K.; Kobashi, S.; Hata, Y. Biometric personal authentication by one step foot pressure distribution change by fuzzy artificial immune system. International Conference on Fuzzy Systems, 2010, pp. 1-6. doi:10.1109/FUZZY.2010.5584216.

58. Ye, H.; Kobashi, S.; Hata, Y.; Taniguchi, K.; Asari, K. Biometric System by Foot Pressure Change Based on Neural Network. 2009 39th International Symposium on Multiple-Valued Logic, 2009, pp. 18-23. doi:10.1109/ISMVL.2009.16.

59. Tivive, F.H.C.; Bouzerdoum, A.; Amin, M.G. A Human Gait Classification Method Based on Radar Doppler Spectrograms. EURASIP J. Adv. Signal Process 2010, 2010, 10:1-10:12. doi:10.1155/2010/389716.

60. Prabhakar, S.; Ivanisov, A.; Jain, A. Biometric recognition: Sensor characteristics and image quality. IEEE Instrumentation Measurement Magazine 2011, 14, 10-16. doi:10.1109/MIM.2011.5773529.

61. Pataky, T.C.; Mu, T.; Bosch, K.; Rosenbaum, D.; Goulermas, J.Y. Gait recognition: highly unique dynamic plantar pressure patterns among 104 individuals. Journal of The Royal Society Interface 2011. doi:10.1098/rsif.2011.0430.

62. Makihara, Y.; Sagawa, R.; Mukaigawa, Y.; Echigo, T.; Yagi, Y., Gait Recognition Using a View Transformation Model in the Frequency Domain. In Computer Vision - ECCV 2006: 9th European Conference on Computer Vision, Graz, Austria, May 7-13, 2006, Proceedings, Part III; Springer Berlin Heidelberg: Berlin, Heidelberg, 2006; pp. 151-163. doi:10.1007/11744078_12.

63. Qian, G.; Zhang, J.; Kidané, A., People Identification Using Gait Via Floor Pressure Sensing and Analysis. In Smart Sensing and Context: Third European Conference, EuroSSC 2008, Zurich, Switzerland, October 29-31, 2008. Proceedings; Springer Berlin Heidelberg: Berlin, Heidelberg, 2008; pp. 83-98. doi:10.1007/978-3-540-88793-5_7. 
64. Qian, G.; Zhang, J.; Kidane, A. People Identification Using Floor Pressure Sensing and Analysis. IEEE Sensors Journal 2010, 10, 1447-1460. doi:10.1109/JSEN.2010.2045158.

65. Hossain, M.A.; Makihara, Y.; Wang, J.; Yagi, Y. Clothing-invariant gait identification using part-based clothing categorization and adaptive weight control. Pattern Recognition 2010, 43, 2281 - 2291. doi:https://doi.org/10.1016/j.patcog.2009.12.020.

66. Moustakas, K.; Tzovaras, D.; Stavropoulos, G. Gait Recognition Using Geometric Features and Soft Biometrics. IEEE Signal Processing Letters 2010, 17, 367-370. doi:10.1109/LSP.2010.2040927.

67. Ioannidis, D.; Tzovaras, D.; Damousis, I.G.; Argyropoulos, S.; Moustakas, K. Gait Recognition Using Compact Feature Extraction Transforms and Depth Information. IEEE Transactions on Information Forensics and Security 2007, 2, 623-630. doi:10.1109/TIFS.2007.902040.

68. Jung, J.W.; Bien, Z.; Lee, S.W.; Sato, T. Dynamic-footprint based person identification using mat-type pressure sensor. Proceedings of the 25th Annual International Conference of the IEEE Engineering in Medicine and Biology Society (IEEE Cat. No.03CH37439), 2003, Vol. 3, pp. 2937-2940. doi:10.1109/IEMBS.2003.1280533.

69. Jung, J.W.; Han, J.S.; Sato, T.; Bien, Z., Unconstrained person recognition method using dynamic partial footprints from floor-type pressure sensor; The Institute of Electronics Engineers of KOREA, 2003; Vol. , pp. $85-88$.

70. Turk, M.; Pentland, A. Eigenfaces for Recognition. Journal of Cognitive Neuroscience 1991, 3, 71-86, [http://dx.doi.org/10.1162/jocn.1991.3.1.71]. PMID: 23964806, doi:10.1162/jocn.1991.3.1.71.

71. Jain, A.K.; Chen, Y.; Demirkus, M. Pores and Ridges: High-Resolution Fingerprint Matching Using Level 3 Features. IEEE Transactions on Pattern Analysis and Machine Intelligence 2007, 29, $15-27$. doi:10.1109/TPAMI.2007.250596.

72. Maltoni, D.; Maio, D.; Jain, A.K.; Prabhakar, S. Handbook of Fingerprint Recognition, 2nd ed.; Springer Publishing Company, Incorporated, 2009.

73. Myers, C.S.; Rabiner, L.R. A comparative study of several dynamic time-warping algorithms for connected-word recognition. The Bell System Technical Journal 1981, 60, 1389-1409. doi:10.1002/j.1538-7305.1981.tb00272.x.

74. Craw, S., Manhattan Distance. In Encyclopedia of Machine Learning; Springer US: Boston, MA, 2010; pp. 639-639. doi:10.1007/978-0-387-30164-8_506.

75. Kumar, A.; Wong, D.C.M.; Shen, H.C.; Jain, A.K. Personal Verification Using Palmprint and Hand Geometry Biometric. Audio- and Video-Based Biometric Person Authentication: 4th International Conference, AVBPA 2003 Guildford, UK, June 9-11, 2003 Proceedings; Kittler, J.; Nixon, M.S., Eds.; Springer Berlin Heidelberg: Berlin, Heidelberg, 2003; pp. 668-678. doi:10.1007/3-540-44887-X_78.

76. Ko, K.; Salamon, W.J. NIST Biometric Image Software (NBIS), 2016.

77. Moorthy, T.N.; Sulaiman, S.F.B. Individualizing characteristics of footprints in Malaysian Malays for person identification from a forensic perspective. Egyptian Journal of Forensic Sciences 2015, 5, 13 - 22. doi:https:/ / doi.org/10.1016/j.ejfs.2014.04.003.

78. Hariharan, M.; Chee, L.S.; Ai, O.C.; Yaacob, S. Classification of Speech Dysfluencies Using LPC Based Parameterization Techniques. Journal of Medical Systems 2012, 36, 1821-1830. doi:10.1007/s10916-010-9641-6.

79. Kumar, A.; Shekhar, S. Personal Identification Using Multibiometrics Rank-Level Fusion. IEEE Transactions on Systems, Man, and Cybernetics, Part C (Applications and Reviews) 2011, 41, 743-752. doi:10.1109/TSMCC.2010.2089516.

80. Kumar, V.D.A.; Ramakrishnan, M.; Jagdeesh, G. A Discrete correlation of Footprint Image aforementioned to Recognition. National Conference on Future Computing, 2012, pp. 5-11.

81. Jia, W.; Cai, H.Y.; Gui, J.; Hu, R.X.; Lei, Y.K.; Wang, X.F. Newborn footprint recognition using orientation feature. Neural Computing and Applications 2012, 21, 1855-1863. doi:10.1007/s00521-011-0530-9.

82. Kumar, V.D.A.; Ramakrishnan, M. Manifold Feature Extraction for Foot Print Image. Indian Journal of Bioinformatics and Biotechnology 2012, 1, 28-31.

83. Kumar, V.D.A.; Ramakrishnan, M. Manifold Feature Extraction for Foot Print Image. Indian Journal of Computer Science and Engineering 2013, 3, 774-778. 
84. Kumar, V.D.A.; Malathi, S.; Kumar, V.D.A.; Kannan, P. Performance Improvement Using an Automation System for Segmentation of Multiple Parametric Features Based on Human Footprint. J. Electr. Eng. Technol. 2015, 10, 1815-1822. doi:10.5370/JEET.2015.10.4.1815.

85. Das, M.; Bandyopadhyay, S.K. Foot Boundary Detection in a Crime Scene. Journal of Scientific and Engineering Research 2016, 3, 24-33.

86. Takeda, T.; Kuramoto, K.; Kobashi, S.; Hata, Y. Fuzzy-logic is precise - Its application to biometric system. Scientia Iranica 2011, 18, 655 - 662. doi:https:/ / doi.org/10.1016/j.scient.2011.05.003.

87. Lu, J.; Tan, Y.P. Uncorrelated discriminant simplex analysis for view-invariant gait signal computing. Pattern Recognition Letters 2010, 31, 382 - 393. doi:https:/ / doi.org/10.1016/j.patrec.2009.11.006.

88. Fernadez, J. The classification of footprints of new born children. Int. Crim. Police Rev. 1953, 64, 52.

89. Jung, J.W.; Sato, T.; Bien, Z. Dynamic footprint-based person recognition method using a hidden markov model and a neural network. International Journal of Intelligent Systems 2004, 19, 1127-1141. doi:10.1002/int.20040.

90. Jang, J.S.R. ANFIS: adaptive-network-based fuzzy inference system. IEEE Transactions on Systems, Man, and Cybernetics 1993, 23, 665-685. doi:10.1109/21.256541.

91. Kohonen, T., Applications. In Self-Organizing Maps; Springer Berlin Heidelberg: Berlin, Heidelberg, 2001; pp. 263-310. doi:10.1007/978-3-642-56927-2_7.

92. Shin, B.S.; Cha, E.Y.; Woo, Y.W.; Klette, R. Segmentation of Scanned Insect Footprints Using ART2 for Threshold Selection. Proceedings of the 2Nd Pacific Rim Conference on Advances in Image and Video Technology; Springer-Verlag: Berlin, Heidelberg, 2007; PSIVT’07, pp. 311-320.

93. Kadyrov, A.; Petrou, M. The Trace transform and its applications. IEEE Transactions on Pattern Analysis and Machine Intelligence 2001, 23, 811-828. doi:10.1109/34.946986.

94. Li, W.; Zhang, D.; Xu, Z. Palmprint Identification by Fourier Transform. International Journal of Pattern Recognition and Artificial Intelligence 2002, 16, 417-432, [http:/ / www.worldscientific.com/doi/pdf/10.1142/S0218001402001757]. doi:10.1142/S0218001402001757.

95. Gonzalez, R.C.; Woods, R.E. Digital Image Processing (3rd Edition); Prentice-Hall, Inc.: Upper Saddle River, NJ, USA, 2006.

96. Al-Dulaimi, K.A. Using Feature Extraction for Human Footprints Recognition. International Journal of Computer Applications 2013, 64.

97. Wayman, J.L., Technical Testing and Evaluation of Biometric Identification Devices. In Biometrics: Personal Identification in Networked Society; Springer US: Boston, MA, 1996; pp. 345-368. doi:10.1007/0-306-47044-6_17.

98. Khokher, R.; Singh, R.C. Footprint-Based Personal Recognition using Scanning Technique. Indian Journal of Science and Technology 2016, 9, 1-10. doi:10.17485/ijst/2016/v9i44/105167. 